\title{
Synthesis of Macroheterocyclic Compounds with a Furan Bridge Possessing Structural Fragments of 1,2,3-Triazoles and Natural Diterpenoids
}

\author{
Yuri V. Kharitonov, ${ }^{\mathrm{a}, \mathrm{b}}$ Makhmut M. Shakirov, ${ }^{\mathrm{a}}$ and Elvira E. Shultsa ${ }^{\mathrm{a}, \mathrm{b}}$ \\ ${ }^{a}$ Novosibirsk Institute of Organic Chemistry, 630090 Novosibirsk, Russia \\ ${ }^{\mathrm{b}}$ Novosibirsk State University, 630090 Novosibirsk, Russia \\ ${ }^{\circledR}$ Corresponding authorE-mail: schultz@nioch.nsc.ru
}

Derivatives of natural diterpenoid lambertianic acid containing alkyne and dialkyne substituent in the furan ring were obtained. 1,2,3-Triazole-incarporated furan bridged macrocycles have been prepared by 1,3-dipolar cycloaddition of methyl 15,16-bis [(prop-2-yn-1-yloxy)methyl]lambertianate with various diazides in the presence of Cu(II)/sodium ascorbate in methylene chloride/water reaction medium.

Keywords: Lambertianic acid, labdanoid alkynes, azides, click chemistry, macroheterocycles.

\section{Синтез макрогетероциклических соединений с фурановым мостиком, содержащих фрагменты 1,2,3-триазолов и природных Аитерпеноидов}

\author{
Ю. В. Харитонов, ${ }^{\mathrm{a}, \mathrm{b}}$ М. М. Шакиров, ${ }^{a}$ Э. Э. Шульц

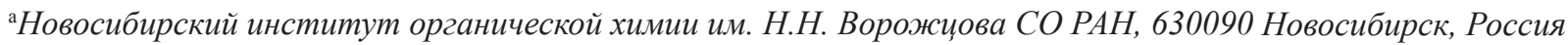 \\ ${ }^{\mathrm{b}}$ Новосибирский национальный исследовательский государственный университет, 630090 Новосибирск, Россия \\ @E-mail: schultz@nioch.nsc.ru
}

\begin{abstract}
Получены производные дитерпеноида ламбертиановой кислоты, содержащие ацетиленовые заместители в фурановом кольце. Реакцией 1,3-диполярного ичиклоприсоединения метил-15,16-бис[(проп-2-ин-1-илокси) метил]ламбертианата с различными диазидами в присутствии Си(II)/аскорбат натрия в системе хлористый метилен-вода синтезированы триазолсодержащие макрогетерочикль с фурановым мостиком.
\end{abstract}

Ключевые слова: Ламбертиановая кислота, лабданоидные алкины, азиды, СuАAC реакция, макрогетероциклы. 


\section{Introduction}

Natural and synthetic macrocyclic molecules are interesting compounds for various application in supramolecular chemistry, ${ }^{[1]}$ material science ${ }^{[2]}$ and in the biological science. [3] Owing to the prominent characteristics, such as chiral rigid skeletons, multiplete chemical reaction sites and unique amphiphilicity steroidal compounds (especially bile acid and its derivatives) are increasingly used as building blocks in supramolecular chemistry, especially in recognition and assembly, ${ }^{[4]}$ because their special characteristics offer a diversity of spacer, different sized cavities, varied binding sites, and unique spatial structure arrangement, as well as biological activity and biocompatibility. ${ }^{[5]}$ With similar structural properties as the biogenic homologue of steroids, triterpenoids (18 $\beta$-glycyrrhetinic acid, oleanolic acid) have also started to emerge in recognition and assembly systems, thus attracting more and more attention from organic and material scientists. ${ }^{[6-8]}$ Among the synthesized macrocyclic steroidal dimers the compounds with good inverse recognition properties, ${ }^{[9]}$ as well as cytotoxic hormone receptor modulating activities ${ }^{[10]}$ were obtained. Glycyrrhetinic acidtweezer receptors with excellent selectivity and affinity for $\mathrm{Hg}^{2+}$ ion, ${ }^{[11]}$ and stable cyclic dimer based on oleanolic acid with remarkable binding ability to $\mathrm{F}^{-}$ion ${ }^{[7]}$ were reported. In the series of macrocyclic diterpenoids from isosteviol ${ }^{[12,13]}$ and steviol, ${ }^{[14]}$ compounds with excellent antituberculosis activity were found. ${ }^{[14]}$ Macrocyclic derivatives of diterpenoid paclitaxel exhibited cytotoxicity against pancreatic cell lines expressing multidrug-resistant genes. ${ }^{[15]}$

In recent years, $\mathrm{Cu}^{\mathrm{I}}$ catalyzed azide-alkyne cycloaddition $(\mathrm{CuAAC})$ received much attention as a method for construction of steroid/triterpenoid-based functional molecules. In view of the specific chemical properties of the formed by this method 1,4-disubstituted 1,2,3-triazoles (stable to metabolic degradation, capable of hydrogen bonding, favorable in the solubility and binding of bimolecular targets because of its relative planarity and strong dipole characteristics ${ }^{[16]}$ ) triazole connecting macrocycles represent a challenging class of molecules with promising therapeutic potential.

During our previous investigation we demonstrated the possibility to synthesize the macrocyclic compounds based on labdane diterpenoids ${ }^{[17]}$ using $\mathrm{CuAAC}$ reaction. In this work we describe the synthesis of compounds, possessing structural fragments of 1,2,3-triazoles and labdanoids with a furan bridge via the CuAAC reaction of a new diacetylenic derivatives - methyl-15,16-epoxy-15,16bis(propinyloxymethyl)labdatriene with diazides.

The development of approaches to macroheterocyclic compounds with a furan bridge produce independent interest because of the high cytotoxicity of macrocyclic diterpenes and $\mathrm{C}_{4}$-norditerpenes of 14-membered cembranoids and polycyclic cembranoids. ${ }^{[18]}$

\section{Experimental}

NMR spectra were acquired on Bruker AV-400 $\left({ }^{1} \mathrm{H}\right.$ : $\left.400.13 \mathrm{MHz},{ }^{13} \mathrm{C}: 100.78 \mathrm{MHz}\right)$ or Bruker AV-600 ( ${ }^{1} \mathrm{H}: 600.30 \mathrm{MHz}$, ${ }^{13} \mathrm{C}$ : $150.95 \mathrm{MHz}$ ) (Bruker BioSpin $\mathrm{GmbH}$, Rheinstetten, Germany) instruments, using tetramethylsilane (TMS) as an internal standard. In the description of the ${ }^{1} \mathrm{H}$ and ${ }^{13} \mathrm{C}$-NMR spectra, the labdane skeleton atoms numeration system given in structure $\mathbf{1}$ was used. The IR spectra were recorded by means of the $\mathrm{KBr}$ pellet technique on a Bruker Vector-22 spectrometer. The UV spectra were obtained on an HP 8453 UV-Vis spectrometer (HewlettPackard, Waldbronn, Germany). Mass spectra were recorded on a DFS spectrometer (Thermo Scientific, evaporator temperature $240-270{ }^{\circ} \mathrm{C}$ ). The melting points were determined on a Stuart SMF-38 melting point apparatus (Bibby Scientific, Staffordshire, UK) and are uncorrected. Elemental analysis was carried out on a Carlo-Erba 1106 analysis instrument. Molecular weights were determined on a VP Osmometer K 7000 (Knauer, Germany). The optical rotation was measured on a polarimeter PolAAr3005 in ethanol at $20-25{ }^{\circ} \mathrm{C}$. Elemental, spectral and analytical investigations were carried out at Collective Chemical Service center of Siberian Branch of the Russian Academy of Sciences.

Reaction products were isolated by column chromatography on silica gel $60(0.063-0.200 \mathrm{~mm}$, Merck KGaA) and eluted with chloroform and chloroform-ethanol (100:1; to 25:1). The reaction progress and the purity of the obtained compounds were monitored by TLC on Silufol UV-254 plates (detection under UV light or by spraying with a $10 \%$ aqueous solution of $\mathrm{H}_{2} \mathrm{SO}_{4}$, followed by heating to $100{ }^{\circ} \mathrm{C}$ ).

Lambertianic acid $\mathbf{1}$ was isolated from the soft resin of Siberian pine Pinus sibirica R. Mayr by known method. ${ }^{[19]}$ Methyl16-formyllabdatrienoate $2,{ }^{[20]}$ methyl 16-(propynyloxymethyl) lambertianate $3,{ }^{[17]}$ diazidopentane $4,{ }^{[2]]} 1,10$-diazidodecane $5,{ }^{[22]}$ 1-azido-2-(2-azidoethoxy)ethane $\mathbf{6},{ }^{[23]}$ and 1,2-bis(2- azidoethoxy) ethane $7^{[23]}$ are known compounds and were prepared by the reported methods. Chemicals used $-\mathrm{POCl}_{3}, \mathrm{NaBH}_{4}, \mathrm{NaH}, 80 \%$ solution of propargyl bromide in $\mathrm{PhMe}, \mathrm{CuSO}_{4} \cdot 5 \mathrm{H}_{2} \mathrm{O}$ - were purchased from Sigma-Aldrich (St. Louis, MO, USA) or Alfa Aesar (GmbH, Karlsruhe, Germany). Solvents (dichloromethane, acetonitrile, $\mathrm{DMF}, \mathrm{MeOH}, i$-propanol) were purified by standard methods and distilled in a stream of argon just before use.

(1S,4aR,5S,8aR)-Methyl-5-(2-\{5-formyl-2-[(prop-2-yn-1-yloxy) methyl]furan-3-yl\}ethyl)-1,4a-dimethyl-6-methylenedecahydronaphthalene-1-carboxylate (8). Compound 3 (1.00 g, $2.51 \mathrm{mmol})$ was dissolved in $15 \mathrm{~mL}$ of dimethylformamide, phosphoryl chloride $(0.45 \mathrm{~mL}, 5.02 \mathrm{mmol})$ was added dropwise under stirring at $20^{\circ} \mathrm{C}$, and the mixture was left to stand for $48 \mathrm{~h}$ at $20^{\circ} \mathrm{C}$. The mixture was then poured into ice water $(40 \mathrm{~mL})$, a saturated aqueous solution of sodium acetate $(20 \mathrm{~mL})$ was added, the organic phase was separated, and the aqueous phase was extracted with chloroform $(3 \times 30 \mathrm{~mL})$. The combined extracts were washed with $5 \%$ aqueous solution of sodium carbonate $(3 \times 30 \mathrm{~mL})$, dried over $\mathrm{MgSO}_{4}$, filtered and evaporated under reduced pressure. The residue was subjected to chromatography on silica gel (petroleum ether-diethyl ether, 4:1) to isolate $0.98 \mathrm{~g}(100 \%)$ of compound $\mathbf{8}$ as an oily substance. Mass spectrum, $m / z\left(I_{\text {rel }}, \%\right): 426(7.8), 358(21), 357$ (87), $325(11), 311$ (13), 310 (19), 297 (36), 189 (28), 187 (10), 181 (11), 175 (17), 161 (10), 149 (16), 133 (12), 123 (13), 122 (11), 121 (100), 199 (19), 109 (16), 107 (27), 105 (20), 95 (15), 93 (25), 91 (30), 81 (32), 79 (21), 77 (13), 67 (15), 55 (17), 41 (18). MS (EI, $70 \mathrm{eV)}$ found: 426.2403. $\mathrm{C}_{26} \mathrm{H}_{34} \mathrm{O}_{5}$. calcd. 426.2401. UV (EtOH) $\lambda_{\max } \mathrm{nm}$ (lge): 289 (4.04). IR $v \mathrm{~cm}^{-1}: 669 \mathrm{w}, 756 \mathrm{~m}, 891 \mathrm{w}, 988 \mathrm{w}, 1028 \mathrm{w}, 1078 \mathrm{~m}, 1155 \mathrm{~s}, 1229$ m, 1317 w, 1352 w, 1381 w, 1447 m, 1466 m, 1528 m, 1645 w, 1684 w, 1720 s, 2118 m, 2847 m, 2872 m, 2945 s, 3078 w, 3269 m, 3302 w, 3424 w. ${ }^{1} \mathrm{H}$ NMR $\left(\mathrm{CDCl}_{3}, 298 \mathrm{~K}\right) \delta_{\mathrm{H}} \mathrm{ppm}: 9.55(1 \mathrm{H}, \mathrm{s}, \mathrm{CHO})$, $7.06\left(1 \mathrm{H}, \mathrm{s}, \mathrm{C}^{14} \mathrm{H}\right), 4.87\left(1 \mathrm{H}, \mathrm{s}, \mathrm{C}^{17} \mathrm{H}\right), 4.53\left(1 \mathrm{H}, \mathrm{s}, \mathrm{C}^{17} \mathrm{H}\right), 4.50(2 \mathrm{H}$, $\left.\mathrm{s}, \mathrm{C}^{1} \mathrm{H}_{2}\right), 4.13\left(2 \mathrm{H}, \mathrm{d}, J=2.4 \mathrm{~Hz}, \mathrm{C}^{3} \mathrm{H}_{2}\right), 3.55\left(3 \mathrm{H}, \mathrm{s}, \mathrm{OCH}_{3}\right), 2.57$ $\left(1 \mathrm{H}, \mathrm{m}, \mathrm{C}^{12} \mathrm{H}\right), 2.45\left(1 \mathrm{H}, \mathrm{t}, J=2.4 \mathrm{~Hz}, \mathrm{C}^{5} \mathrm{H}\right), 2.37(1 \mathrm{H}, \mathrm{t} . \mathrm{d}, J=12.4 \mathrm{~Hz}$, $\left.J=3.1 \mathrm{~Hz}, \mathrm{C}^{7} \mathrm{H}\right), 2.31\left(1 \mathrm{H}, \mathrm{m}, \mathrm{C}^{12} \mathrm{H}\right), 2.10\left(1 \mathrm{H}, \mathrm{d} . \mathrm{m}, J=12.9 \mathrm{~Hz}, \mathrm{C}^{3} \mathrm{H}\right)$, $1.94\left(1 \mathrm{H}, \mathrm{m}, \mathrm{C}^{6} \mathrm{H}\right), 1.67-1.85\left(5 \mathrm{H}, \mathrm{m}, \mathrm{C}^{1} \mathrm{H}, \mathrm{C}^{2} \mathrm{H}, \mathrm{C}^{6} \mathrm{H}, \mathrm{C}^{7} \mathrm{H}, \mathrm{C}^{11} \mathrm{H}\right)$, $1.57\left(1 \mathrm{H}, \mathrm{m}, \mathrm{C}^{11} \mathrm{H}\right), 1.53\left(1 \mathrm{H}, \mathrm{m}, \mathrm{C}^{9} \mathrm{H}\right), 1.44\left(1 \mathrm{H}, \mathrm{m}, \mathrm{C}^{2} \mathrm{H}\right), 1.21$ $\left(1 \mathrm{H}\right.$, d.d, $\left.J=12.4 \mathrm{~Hz}, J=2.7 \mathrm{~Hz}, \mathrm{C}^{5} \mathrm{H}\right), 1.12\left(3 \mathrm{H}, \mathrm{s}, \mathrm{C}^{19} \mathrm{H}_{3}\right), 0.96(1 \mathrm{H}$, d.t, $\left.J=13.3 \mathrm{~Hz}, J=4.0 \mathrm{~Hz}, \mathrm{C}^{3} \mathrm{H}\right), 0.91(1 \mathrm{H}$, d.t, $J=13.3 \mathrm{~Hz}, J=3.5 \mathrm{~Hz}$, $\left.\mathrm{C}^{1} \mathrm{H}\right), 0.45\left(3 \mathrm{H}, \mathrm{s}, \mathrm{C}^{20} \mathrm{H}_{3}\right) \cdot{ }^{13} \mathrm{C} \mathrm{NMR}\left(\mathrm{CDCl}_{3}, 298 \mathrm{~K}\right) \delta_{\mathrm{C}} \mathrm{ppm}: 177.9$ 
(CHO), 177.6 $\left(\mathrm{C}^{18}\right), 151.9\left(\mathrm{C}^{16}\right)^{*}, 151.8\left(\mathrm{C}^{15}\right)^{*}, 147.6\left(\mathrm{C}^{8}\right), 127.3$ $\left(\mathrm{C}^{13}\right), 122.0\left(\mathrm{C}^{14}\right), 106.5\left(\mathrm{C}^{17}\right), 78.9\left(\mathrm{C}^{4}\right), 75.3\left(\mathrm{C}^{5}\right), 61.3\left(\mathrm{C}^{1}\right), 57.5$ $\left(\mathrm{C}^{3}\right), 56.2\left(\mathrm{C}^{5}\right), 54.9\left(\mathrm{C}^{9}\right), 51.1\left(\mathrm{OCH}_{3}\right), 44.2\left(\mathrm{C}^{4}\right), 40.2\left(\mathrm{C}^{10}\right), 39.0$ $\left(\mathrm{C}^{1}\right), 38.6\left(\mathrm{C}^{7}\right), 38.1\left(\mathrm{C}^{3}\right), 28.7\left(\mathrm{C}^{19}\right), 26.2\left(\mathrm{C}^{6}\right), 24.4\left(\mathrm{C}^{11}\right), 22.9\left(\mathrm{C}^{12}\right)$, $19.9\left(\mathrm{C}^{2}\right), 12.6\left(\mathrm{C}^{20}\right)$

(1S,4aR,5S,8aR)-Methyl-5-(2-\{5-(hydroxymethyl)-2-[(prop-2yn-1-yloxy)methyl]furan-3-yl'sethyl)-1,4a-dimethyl-6-methylenedecahydronaphthalene-1-carboxylate (9). $\mathrm{NaBH}_{4}(0.89 \mathrm{~g}, 23.44 \mathrm{mmol})$ was added portion wise to a solution of aldehyde $8(1.00 \mathrm{~g}, 2.34 \mathrm{mmol})$ in $15 \mathrm{~mL}$ of $i$-propanol under stirring at $20^{\circ} \mathrm{C}$. After stirring for $24 \mathrm{~h}$ at $20^{\circ} \mathrm{C}$ the mixture was diluted with water, and extracted with chloroform $(3 \times 30 \mathrm{~mL})$. The combined extract was washed with water $(3 \times 30 \mathrm{~mL})$, dried over $\mathrm{MgSO}_{4}$, filtered and evaporated. The residue was subjected to column chromatography on silica gel (petroleum ether-diethyl ether, $2: 1)$ to isolate $1.00 \mathrm{~g}(97 \%)$ of compound 9 as an oily substance. [ $\alpha$ ] D $+40.39^{\circ}$ (c 3.61; $\left.\mathrm{CHCl}_{3}\right)$. Mass spectrum, $\mathrm{m} / z\left(I_{\text {rel }}, \%\right): 428(4), 372$ (12), 357 (24), 341 (12), 249 (11), 193 (13), 189 (36), $188(10), 181$ (15), 180 (14), 178 (22), 173 (15), 161 (13), 150 (24), 149 (19), 147 (10), 133 (16), 133 (11), 125 (15), 124 (45), 123 (10), 122 (13), 121 (100), 199 (22), 109 (22), 107 (31), 105 (27), 95 (20), 93 (32), 91 (32), 83 (10), 81 (44), 79 (27), 77 (17), 67 (21), 55 (27), 53 (12), 43 (15), 41 (26), 39 (15). MS (EI, $70 \mathrm{eV}$ ) found: 428.2551. $\mathrm{C}_{26} \mathrm{H}_{36} \mathrm{O}_{5}$. calcd. 428.2557. UV (EtOH) $\lambda_{\text {max }} \mathrm{nm}$ (lge): 228 (3.84), 289 (3.31). IR (KBr) $v \mathrm{~cm}^{-1}: 667 \mathrm{w}$, $756 \mathrm{~m}, 889 \mathrm{w}, 984 \mathrm{w}, 1070 \mathrm{~m}, 1155 \mathrm{~s}, 1229 \mathrm{~m}, 1383 \mathrm{w}, 1447 \mathrm{~m}, 1466$ m, 1528 m, 1643 w, 1682 w, 1722 s, 1761 m, 2118 m, 2849 m, 2874 m, 2945 s, 3078 w, 3306 w, 3433 w. ${ }^{1} \mathrm{H} \mathrm{NMR}\left(\mathrm{CDCl}_{3}, 298 \mathrm{~K}\right) \delta_{\mathrm{H}} \mathrm{ppm}$ : $6.14\left(1 \mathrm{H}, \mathrm{s}, \mathrm{C}^{14} \mathrm{H}\right), 4.88\left(1 \mathrm{H}, \mathrm{s}, \mathrm{C}^{17} \mathrm{H}\right), 4.56\left(1 \mathrm{H}, \mathrm{s}, \mathrm{C}^{17} \mathrm{H}\right), 4.54(2 \mathrm{H}, \mathrm{s}$, $\left.\mathrm{C}^{\mathrm{l}^{\prime}} \mathrm{H}_{2}\right), 4.44\left(2 \mathrm{H}, \mathrm{s}, \mathrm{C}^{\mathrm{l}^{\prime \prime}} \mathrm{H}_{2}\right), 4.11\left(2 \mathrm{H}, \mathrm{d}, J=2.3 \mathrm{~Hz}, \mathrm{C}^{3} \mathrm{H}_{2}\right), 3.59(3 \mathrm{H}, \mathrm{s}$, $\left.\mathrm{OCH}_{3}\right), 2.52\left(1 \mathrm{H}, \mathrm{m}, \mathrm{C}^{12} \mathrm{H}\right), 2.43\left(1 \mathrm{H}, \mathrm{t}, J=2.3 \mathrm{~Hz}, \mathrm{C}^{5} \mathrm{H}\right), 2.40(1 \mathrm{H}, \mathrm{m}$, $\left.\mathrm{C}^{7} \mathrm{H}\right), 2.25\left(1 \mathrm{H}, \mathrm{m}, \mathrm{C}^{12} \mathrm{H}\right), 2.13\left(1 \mathrm{H}, \mathrm{d} . \mathrm{m}, J=13.2 \mathrm{~Hz}, \mathrm{C}^{3} \mathrm{H}\right), 1.96(1 \mathrm{H}$, $\left.\mathrm{m}, \mathrm{C}^{6} \mathrm{H}\right), 1.67,1.76,1.82,1.88\left(5 \mathrm{H}\right.$, all $\left.\mathrm{m}, \mathrm{C}^{1} \mathrm{H}, \mathrm{C}^{2} \mathrm{H}, \mathrm{C}^{6} \mathrm{H}, \mathrm{C}^{7} \mathrm{H}, \mathrm{C}^{11} \mathrm{H}\right)$, $1.57\left(2 \mathrm{H}, \mathrm{m}, \mathrm{C}^{11} \mathrm{H}, \mathrm{C}^{9} \mathrm{H}\right), 1.47\left(1 \mathrm{H}, \mathrm{m}, \mathrm{C}^{2} \mathrm{H}\right), 1.26(1 \mathrm{H}, \mathrm{d} . \mathrm{d}, J=12.4 \mathrm{~Hz}$, $\left.J=2.8 \mathrm{~Hz}, \mathrm{C}^{5} \mathrm{H}\right), 1.15\left(3 \mathrm{H}, \mathrm{s}, \mathrm{C}^{19} \mathrm{H}_{3}\right), 1.00(1 \mathrm{H}$, d.t, $J=13.2 \mathrm{~Hz}, J=3.9 \mathrm{~Hz}$, $\left.\mathrm{C}^{3} \mathrm{H}\right), 0.96\left(1 \mathrm{H}\right.$, d.t, $\left.J=14.2 \mathrm{~Hz}, J=2.7 \mathrm{~Hz}, \mathrm{C}^{1} \mathrm{H}\right), 0.47\left(3 \mathrm{H}, \mathrm{s}, \mathrm{C}^{20} \mathrm{H}_{3}\right)$. ${ }^{13} \mathrm{C}$ NMR $\left(\mathrm{CDCl}_{3}, 298 \mathrm{~K}\right) \delta_{\mathrm{C}} \mathrm{ppm}: 177.6\left(\mathrm{C}^{18}\right), 153.8\left(\mathrm{C}^{16}\right), 147.7\left(\mathrm{C}^{8}\right)$, $146.0\left(\mathrm{C}^{15}\right), 125.9\left(\mathrm{C}^{13}\right), 109.4\left(\mathrm{C}^{14}\right), 106.4\left(\mathrm{C}^{17}\right), 79.4\left(\mathrm{C}^{4}\right), 74.7\left(\mathrm{C}^{5}\right)$, $61.1\left(\mathrm{C}^{1}\right), 57.5\left(\mathrm{C}^{\prime \prime}\right), 56.6\left(\mathrm{C}^{3}\right), 56.1\left(\mathrm{C}^{5}\right), 54.9\left(\mathrm{C}^{9}\right), 51.0\left(\mathrm{OCH}_{3}\right), 44.2$ $\left(\mathrm{C}^{4}\right), 40.1\left(\mathrm{C}^{10}\right), 38.9\left(\mathrm{C}^{1}\right), 38.6\left(\mathrm{C}^{7}\right), 38.1\left(\mathrm{C}^{3}\right), 28.7\left(\mathrm{C}^{19}\right), 26.2\left(\mathrm{C}^{6}\right)$, $24.5\left(\mathrm{C}^{11}\right), 23.2\left(\mathrm{C}^{12}\right), 19.8\left(\mathrm{C}^{2}\right), 12.5\left(\mathrm{C}^{20}\right)$

By carrying out this reaction in methanol (1S,4aR, 5S, $8 a R)$ methyl 5-(2-\{5-(dimethoxymethyl)-2-[(prop-2-yn-1-yloxy)methyl] furan-3-yl\}ethyl)-1,4a-dimethyl-6-methylenedecahydronaphthalene1-carboxylate (10) was obtained in the yield $98 \%$. Oily substance. ${ }^{1} \mathrm{H}$ NMR $\left(\mathrm{CDCl}_{3}, 298 \mathrm{~K}\right) \delta_{\mathrm{H}} \mathrm{ppm}: 6.24\left(1 \mathrm{H}, \mathrm{s}, \mathrm{C}^{14} \mathrm{H}\right), 5.32(1 \mathrm{H}, \mathrm{s}$, $\left.\mathrm{C} H\left(\mathrm{OCH}_{3}\right)_{2}\right), 4.84\left(1 \mathrm{H}, \mathrm{s}, \mathrm{C}^{17} \mathrm{H}\right), 4.53\left(1 \mathrm{H}, \mathrm{s}, \mathrm{C}^{17} \mathrm{H}\right), 4.40(2 \mathrm{H}, \mathrm{s}$, $\left.\mathrm{C}^{1} \mathrm{H}_{2}\right), 4.05\left(2 \mathrm{H}, \mathrm{d}, J=2.2 \mathrm{~Hz}, \mathrm{C}^{3} \mathrm{H}_{2}\right), 3.54\left(3 \mathrm{H}, \mathrm{s}, \mathrm{OCH}_{3}\right), 3.29(6 \mathrm{H}$, $\left.\mathrm{s}, \mathrm{CH}\left(\mathrm{OCH}_{3}\right)_{2}\right), 2.49\left(1 \mathrm{H}, \mathrm{m}, \mathrm{C}^{12} \mathrm{H}\right), 2.39\left(1 \mathrm{H}, \mathrm{t}, J=2.2 \mathrm{~Hz}, \mathrm{C}^{5} \mathrm{H}\right), 2.35$ $\left(1 \mathrm{H}, \mathrm{m}, \mathrm{C}^{7} \mathrm{H}\right), 2.21\left(1 \mathrm{H}, \mathrm{m} . \mathrm{C}^{12} \mathrm{H}\right), 2.09\left(1 \mathrm{H}, \mathrm{d} . \mathrm{m}, J=12.6 \mathrm{~Hz}, \mathrm{C}^{3} \mathrm{H}\right)$, $1.92\left(1 \mathrm{H}, \mathrm{m}, \mathrm{C}^{6} \mathrm{H}\right), 1.65,1.74,1.85\left(5 \mathrm{H}\right.$, all $\mathrm{m}, \mathrm{C}^{1} \mathrm{H}, \mathrm{C}^{2} \mathrm{H}, \mathrm{C}^{6} \mathrm{H}, \mathrm{C}^{7} \mathrm{H}$, $\left.\mathrm{C}^{11} \mathrm{H}\right), 1.53\left(2 \mathrm{H}, \mathrm{m}, \mathrm{C}^{11} \mathrm{H}, \mathrm{C}^{9} \mathrm{H}\right), 1.42\left(1 \mathrm{H}, \mathrm{m}, \mathrm{C}^{2} \mathrm{H}\right), 1.21(1 \mathrm{H}$, d.d, $\left.J=12.2 \mathrm{~Hz}, J=2.7 \mathrm{~Hz}, \mathrm{C}^{5} \mathrm{H}\right), 1.11\left(3 \mathrm{H}, \mathrm{s}, \mathrm{C}^{19} \mathrm{H}_{3}\right), 0.95(1 \mathrm{H}$, d.t, $J=13.3$ $\left.\mathrm{Hz}, J=3.8 \mathrm{~Hz}, \mathrm{C}^{3} \mathrm{H}\right), 0.91\left(1 \mathrm{H}\right.$, d.t, $\left.J=14.2 \mathrm{~Hz}, J=3.5 \mathrm{~Hz}, \mathrm{C}^{1} \mathrm{H}\right), 0.43$ $\left(3 \mathrm{H}, \mathrm{s}, \mathrm{C}^{20} \mathrm{H}_{3}\right) \cdot{ }^{13} \mathrm{C}$ NMR $\left(\mathrm{CDCl}_{3}, 298 \mathrm{~K}\right) \delta_{\mathrm{C}} \mathrm{ppm}: 177.4\left(\mathrm{C}^{18}\right), 146.2$ $\left(\mathrm{C}^{8}\right), 150.3\left(\mathrm{C}^{16}\right), 147.6\left(\mathrm{C}^{15}\right), 125.5\left(\mathrm{C}^{13}\right), 111.1\left(\mathrm{C}^{14}\right), 106.3\left(\mathrm{C}^{17}\right)$, $97.8\left[\mathrm{CH}\left(\mathrm{OCH}_{3}\right)_{2}\right], 79.3\left(\mathrm{C}^{4}\right), 74.6\left(\mathrm{C}^{5^{\prime}}\right), 60.9\left(\mathrm{C}^{1}\right), 56.5\left(\mathrm{C}^{3^{\prime}}\right), 56.0$ $\left(\mathrm{C}^{5}\right), 54.8\left(\mathrm{C}^{9}\right), 52.6,52.7\left(\mathrm{OCH}_{3}\right), 50.9\left(\mathrm{OCH}_{3}\right), 44.0\left(\mathrm{C}^{4}\right), 39.9\left(\mathrm{C}^{10}\right)$, $38.9\left(\mathrm{C}^{1}\right), 38.5\left(\mathrm{C}^{7}\right), 37.9\left(\mathrm{C}^{3}\right), 28.6\left(\mathrm{C}^{19}\right), 26.1\left(\mathrm{C}^{6}\right), 24.4\left(\mathrm{C}^{11}\right), 23.2$ $\left(\mathrm{C}^{12}\right), 19.7\left(\mathrm{C}^{2}\right), 12.4\left(\mathrm{C}^{20}\right)$.

(1S,4aR,5S,8aR)-Methyl-5-(2-\{2,5-bis[(prop-2-yn-1-yloxy) methyl]furan-3-yl; ethyl)-1,4a-dimethyl-6-methylenedecahydronaphthalene-1-carboxylate (11). A) To a stirred solution of compound $9(1.00 \mathrm{~g}, 2.2 \mathrm{mmol})$ in $10 \mathrm{~mL}$ of acetonitrile a dispersion of sodium hydride in mineral oil $(0.17 \mathrm{~g}, 4.3 \mathrm{mmol})$ was added at $0{ }^{\circ} \mathrm{C}$ portionwise. The mixture was stirred for $30 \mathrm{~min}$, and a solution of propargyl bromide in toluene $(0.48 \mathrm{~mL}, 4.3 \mathrm{mmol})$ was added. The reaction mixture was warmed to ambient temperature and stirred for additional 4 $\mathrm{h}$, then poured on $50 \mathrm{~g}$ of ice, and extracted with chloroform $(3 \times 50 \mathrm{~mL})$. The combined extracts were washed with water $(3 \times 50 \mathrm{~mL})$, dried over
$\mathrm{MgSO}_{4}$, filtered and evaporated. The residue was subjected to column chromatography on silica gel. Eluting with a mixture of petroleum ether-diethyl ether, $10: 1$ gave $0.73 \mathrm{~g}(73 \%)$ of compound 11 as an oily substance. B) To a stirred solution of compound $9(1.00 \mathrm{~g}, 2.2 \mathrm{mmol})$ in $10 \mathrm{~mL}$ of DMF a dispersion of sodium hydride in mineral oil $(0.17 \mathrm{~g}$, $4.3 \mathrm{mmol}$ ) was added at $0{ }^{\circ} \mathrm{C}$. The mixture was stirred for $30 \mathrm{~min}$, and a solution of propargyl bromide in toluene $(0.48 \mathrm{~mL}, 4.3 \mathrm{mmol})$ was added. The reaction mixture was warmed to ambient temperature and stirred for additional $4 \mathrm{~h}$ then poured on $50 \mathrm{~g}$ of ice, and extracted with chloroform $(3 \times 50 \mathrm{~mL})$. The combined extracts were washed with water $(7 \times 50 \mathrm{~mL})$, dried over $\mathrm{MgSO}_{4}$ and evaporated. By column chromatography of the residue on silica gel (petroleum ether-diethyl ether, 10:1 as an eluent) compounds $\mathbf{1 1}(0.37 \mathrm{~g}$, yield $34 \%)$ and $\mathbf{1 2}(0.24 \mathrm{~g}$, yield $23 \%$ ) were successively isolated.

Compound 11. $[\alpha]_{\mathrm{D}}+16.00^{\circ}\left(\mathrm{c} 0.20 ; \mathrm{CHCl}_{3}\right)$. Mass spectrum, $\mathrm{m} / \mathrm{z}\left(I_{\text {rel }}, \%\right): 466$ (5), 411 (18), 397 (26), 341 (17), 231 (23), 218 (16), 216 (29), 189 (27), 181 (14), 173 (20), 162 (40), 161 (14), 149 (16), 133 (18), 131 (14), 121 (100), 119 (23), 117 (13), 109 (26), 107 (33), 105 (32), 97 (15), 95 (26), 93 (31), 91 (38), 85 (41), 83 (64), 81 (46), 79 (28), 77 (19), 71 (14), 69 (23), 67 (23), 57 (20), 55 (34), 53 (13), 46 (16), 43 (19), 41 (40), 39 (35). MS (EI, 70 eV) found: 466.2711. $\mathrm{C}_{29} \mathrm{H}_{38} \mathrm{O}_{5}$. calcd. 466.2714. UV (EtOH) $\lambda_{\max } \mathrm{nm}$ $(\lg \varepsilon): 230$ (4.00). IR $(\mathrm{KBr}) v \mathrm{~cm}^{-1}: 635 \mathrm{w}, 671 \mathrm{w}, 822 \mathrm{~m}, 889 \mathrm{w}$, $988 \mathrm{w}, 1030 \mathrm{w}, 1074 \mathrm{~m}, 1155 \mathrm{~s}, 1229 \mathrm{~m}, 1352 \mathrm{w}, 1383 \mathrm{w}, 1445 \mathrm{~m}$, 1464 m, 1643 w, 1684 w, 1722 s, 1761 m, 2116 m, 2849 m, 2945 s, $3078 \mathrm{w}, 3292$ w. ${ }^{1} \mathrm{H}$ NMR $\left(\mathrm{CDCl}_{3}, 298 \mathrm{~K}\right) \delta_{\mathrm{H}} \mathrm{ppm}$ : $6.20(1 \mathrm{H}, \mathrm{s}$, $\left.\mathrm{C}^{14} \mathrm{H}\right), 4.86\left(1 \mathrm{H}, \mathrm{s}, \mathrm{C}^{17} \mathrm{H}\right), 4.54\left(1 \mathrm{H}, \mathrm{s}, \mathrm{C}^{17} \mathrm{H}\right), 4.47\left(2 \mathrm{H}, \mathrm{s}, \mathrm{C}^{1} \mathrm{H}_{2}\right)^{*}$, $4.42\left(2 \mathrm{H}, \mathrm{s}, \mathrm{C}^{1 "} \mathrm{H}_{2}\right)^{*}, 4.12\left(2 \mathrm{H}, \mathrm{d}, J=2.4 \mathrm{~Hz}, \mathrm{C}^{3} \mathrm{H}_{2}\right)^{* *}, 4.08(2 \mathrm{H}$, $\left.\mathrm{d}, J=2.4 \mathrm{~Hz}, \mathrm{C}^{3} \mathrm{H}_{2}\right)^{* *}, 3.56\left(3 \mathrm{H}, \mathrm{s}, \mathrm{OCH}_{3}\right), 2.50\left(1 \mathrm{H}, \mathrm{m}, \mathrm{C}^{12} \mathrm{H}\right)$, $2.43\left(1 \mathrm{H}, \mathrm{t}, J=2.4 \mathrm{~Hz}, \mathrm{C}^{5} \mathrm{H}\right) *, 2.41\left(1 \mathrm{H}, \mathrm{t}, J=2.4 \mathrm{~Hz}, \mathrm{C}^{5 \prime} \mathrm{H}\right)^{*}, 2.37$ $\left(1 \mathrm{H}, \mathrm{m}, \mathrm{C}^{7} \mathrm{H}\right), 2.24\left(1 \mathrm{H}, \mathrm{m}, \mathrm{C}^{12} \mathrm{H}\right), 2.11\left(1 \mathrm{H}, \mathrm{d} . \mathrm{m}, J=12.4 \mathrm{~Hz}, \mathrm{C}^{3} \mathrm{H}\right)$, $1.93\left(1 \mathrm{H}, \mathrm{m}, \mathrm{C}^{6} \mathrm{H}\right), 1.65,1.74,1.84\left(5 \mathrm{H}\right.$, all $\mathrm{m}, \mathrm{C}^{1} \mathrm{H}, \mathrm{C}^{2} \mathrm{H}, \mathrm{C}^{6} \mathrm{H}$, $\left.\mathrm{C}^{7} \mathrm{H}, \mathrm{C}^{11} \mathrm{H}\right), 1.55\left(2 \mathrm{H}, \mathrm{m}, \mathrm{C}^{11} \mathrm{H}, \mathrm{C}^{9} \mathrm{H}\right), 1.45\left(1 \mathrm{H}, \mathrm{m}, \mathrm{C}^{2} \mathrm{H}\right), 1.23(1 \mathrm{H}$, d.d, $\left.J=12.4 \mathrm{~Hz}, J=3.0 \mathrm{~Hz}, \mathrm{C}^{5} \mathrm{H}\right), 1.13\left(3 \mathrm{H}, \mathrm{s}, \mathrm{C}^{19} \mathrm{H}_{3}\right), 0.98(1 \mathrm{H}$, d.t, $\left.J=13.2 \mathrm{~Hz}, J=4.0 \mathrm{~Hz}, \mathrm{C}^{3} \mathrm{H}\right), 0.93(1 \mathrm{H}$, d.t, $J=13.4 \mathrm{~Hz}, J=3.8 \mathrm{~Hz}$, $\left.\mathrm{C}^{1} \mathrm{H}\right), 0.45\left(3 \mathrm{H}, \mathrm{s}, \mathrm{C}^{20} \mathrm{H}_{3}\right) .{ }^{13} \mathrm{C} \mathrm{NMR}\left(\mathrm{CDCl}_{3}, 298 \mathrm{~K}\right) \delta_{\mathrm{C}} \mathrm{ppm}: 177.6$ $\left(\mathrm{C}^{18}\right), 150.4\left(\mathrm{C}^{16}\right), 147.7\left(\mathrm{C}^{8}\right), 146.8\left(\mathrm{C}^{15}\right), 125.8\left(\mathrm{C}^{13}\right), 111.8\left(\mathrm{C}^{14}\right)$, $\left.106.4\left(\mathrm{C}^{17}\right), 79.4\left(\mathrm{C}^{4}\right) *, 79.2\left(\mathrm{C}^{4^{\prime \prime}}\right)^{*}, 74.8\left(\mathrm{C}^{5^{7}}\right)^{* *}, 74.6\left(\mathrm{C}^{5}\right)\right)^{* *}, 63.2$ $\left(\mathrm{C}^{1}\right)^{*}, 61.1\left(\mathrm{C}^{1^{*}}\right)^{*}, 56.8\left(\mathrm{C}^{3}\right)^{* *}, 56.7\left(\mathrm{C}^{3}\right)^{* *}, 56.1\left(\mathrm{C}^{5}\right), 54.9\left(\mathrm{C}^{9}\right)$, $51.0\left(\mathrm{OCH}_{3}\right), 44.2\left(\mathrm{C}^{4}\right), 40.0\left(\mathrm{C}^{10}\right), 38.9\left(\mathrm{C}^{1}\right), 38.6\left(\mathrm{C}^{7}\right), 38.0\left(\mathrm{C}^{3}\right)$, $28.7\left(\mathrm{C}^{19}\right), 26.2\left(\mathrm{C}^{6}\right), 24.5\left(\mathrm{C}^{11}\right), 23.2\left(\mathrm{C}^{12}\right), 19.8\left(\mathrm{C}^{2}\right), 12.5\left(\mathrm{C}^{20}\right)$.

(1S, 4aR, 5S, 8aR)-Methyl-5-(2-\{5-[(formyloxy)methyl)2-((prop-2-yn-1-yloxy)methyl]furan-3-yl\}ethyl)-1,4a-dimethyl6-methylenedecahydronaphthalene-1-carboxylate (12). Mass spectrum, $m / z\left(I_{\text {rel }} \%\right)$ : $456(1), 357$ (16), 189 (31), 181 (16), 173 (14), 161 (15), 133 (16), 121 (100), 119 (22), 109 (23), 107 (33), 105 (26), 95 (18), 93 (27), 91 (28), 81 (37), 79 (24), 67 (16), 55 (21), 41 (24), 39 (16). MS (EI, $70 \mathrm{eV}$ ) found: 456.2507 . calcd. for $\mathrm{C}_{27} \mathrm{H}_{36} \mathrm{O}_{6} .456 .2506$. UV (EtOH) $\lambda_{\text {max }} \mathrm{nm}(\lg \varepsilon): 228$ (2.98), 284 (2.28). IR (KBr) $v \mathrm{~cm}^{-1}$ : $667 \mathrm{w}, 756 \mathrm{~m}, 891 \mathrm{w}, 934 \mathrm{w}, 986 \mathrm{w}, 1030 \mathrm{~m}, 1074 \mathrm{~m}, 1155 \mathrm{~s}, 1228 \mathrm{~m}$, $1360 \mathrm{~m}, 1383 \mathrm{w}, 1447 \mathrm{~m}, 1464 \mathrm{~m}, 1645 \mathrm{w}, 1722 \mathrm{~s}, 1761 \mathrm{~m}, 2849 \mathrm{~m}$, $2874 \mathrm{~m}, 2946 \mathrm{~s}, 3290 \mathrm{w}, 3429$ w. ${ }^{1} \mathrm{H}$ NMR $\left(\mathrm{CDCl}_{3}, 298 \mathrm{~K}\right) \delta_{\mathrm{H}} \mathrm{ppm}$ : $8.06\left(1 \mathrm{H}, \mathrm{s}, \mathrm{CH}_{2} \mathrm{OCHO}\right), 6.28\left(1 \mathrm{H}, \mathrm{s}, \mathrm{C}^{14} \mathrm{H}\right), 5.07\left(2 \mathrm{H}, \mathrm{s}, \mathrm{CH}_{2} \mathrm{OCHO}\right)$, $4.88\left(1 \mathrm{H}, \mathrm{s}, \mathrm{C}^{17} \mathrm{H}\right), 4.55\left(1 \mathrm{H}, \mathrm{s}, \mathrm{C}^{17} \mathrm{H}\right), 4.44\left(2 \mathrm{H}, \mathrm{s}, \mathrm{C}^{1} \mathrm{H}_{2}\right), 4.12(1 \mathrm{H}$, $\left.\mathrm{d}, J=2.3 \mathrm{~Hz}, \mathrm{C}^{3} \mathrm{H}\right), 4.10\left(1 \mathrm{H}, \mathrm{d}, J=2.3 \mathrm{~Hz}, \mathrm{C}^{3} \mathrm{H}\right), 3.58\left(3 \mathrm{H}, \mathrm{s}, \mathrm{OCH}_{3}\right)$, $2.51\left(1 \mathrm{H}, \mathrm{m}, \mathrm{C}^{12} \mathrm{H}\right), 2.43\left(1 \mathrm{H}, \mathrm{t}, J=2.4 \mathrm{~Hz}, \mathrm{C}^{5} \mathrm{H}\right), 2.40\left(1 \mathrm{H}, \mathrm{m}, \mathrm{C}^{7} \mathrm{H}\right)$, $2.25\left(1 \mathrm{H}, \mathrm{m}, \mathrm{C}^{12} \mathrm{H}\right), 2.13\left(1 \mathrm{H}\right.$, d.m, $\left.J=12.6 \mathrm{~Hz}, \mathrm{C}^{3} \mathrm{H}\right), 1.95(1 \mathrm{H}, \mathrm{m}$, $\left.\mathrm{C}^{6} \mathrm{H}\right), 1.67,1.74,1.78,1.85\left(5 \mathrm{H}\right.$, all $\left.\mathrm{m}, \mathrm{C}^{1} \mathrm{H}, \mathrm{C}^{2} \mathrm{H}, \mathrm{C}^{6} \mathrm{H}, \mathrm{C}^{7} \mathrm{H}, \mathrm{C}^{11} \mathrm{H}\right)$, $1.58\left(2 \mathrm{H}, \mathrm{m}, \mathrm{C}^{11} \mathrm{H}, \mathrm{C}^{9} \mathrm{H}\right), 1.49\left(1 \mathrm{H}, \mathrm{m}, \mathrm{C}^{2} \mathrm{H}\right), 1.25(1 \mathrm{H}$, d.d, $J=12.1$ $\left.\mathrm{Hz}, J=2.5 \mathrm{~Hz}, \mathrm{C}^{5} \mathrm{H}\right), 1.15\left(3 \mathrm{H}, \mathrm{s}, \mathrm{C}^{19} \mathrm{H}_{3}\right), 0.99(1 \mathrm{H}$, d.t, $J=13.4 \mathrm{~Hz}$, $\left.J=4.3 \mathrm{~Hz}, \mathrm{C}^{3} \mathrm{H}\right), 0.95\left(1 \mathrm{H}\right.$, d.t, $\left.J=13.9 \mathrm{~Hz}, J=4.0 \mathrm{~Hz}, \mathrm{C}^{1} \mathrm{H}\right), 0.47(3 \mathrm{H}$, $\left.\mathrm{s}, \mathrm{C}^{20} \mathrm{H}_{3}\right) \cdot{ }^{13} \mathrm{C} \mathrm{NMR}\left(\mathrm{CDCl}_{3}, 298 \mathrm{~K}\right) \delta_{\mathrm{C}} \mathrm{ppm}: 177.6\left(\mathrm{C}^{18}\right), 160.4(\mathrm{CHO})$, $148.2\left(\mathrm{C}^{16}\right), 147.8\left(\mathrm{C}^{8}\right), 147.3\left(\mathrm{C}^{15}\right), 126.1\left(\mathrm{C}^{13}\right), 112.8\left(\mathrm{C}^{14}\right), 106.4\left(\mathrm{C}^{17}\right)$, $79.3\left(\mathrm{C}^{4}\right), 74.8\left(\mathrm{C}^{5^{\prime}}\right), 61.1\left(\mathrm{C}^{1^{\prime}}\right), 57.5\left(\mathrm{CH}_{2}\right), 56.9\left(\mathrm{C}^{3}\right), 56.1\left(\mathrm{C}^{5}\right), 54.9$ $\left(\mathrm{C}^{9}\right), 51.1\left(\mathrm{OCH}_{3}\right), 44.2\left(\mathrm{C}^{4}\right), 40.1\left(\mathrm{C}^{10}\right), 39.0\left(\mathrm{C}^{1}\right), 38.6\left(\mathrm{C}^{7}\right), 38.1\left(\mathrm{C}^{3}\right)$, $28.7\left(\mathrm{C}^{19}\right), 26.2\left(\mathrm{C}^{6}\right), 24.6\left(\mathrm{C}^{11}\right), 23.2\left(\mathrm{C}^{12}\right), 19.9\left(\mathrm{C}^{2}\right), 12.6\left(\mathrm{C}^{20}\right)$.

Reaction of methyl ester (15,16-((prop-2-yn-1-yloxy) methyl)-15,16-epoxy-8(9),13(16), 14-labdatrienoate (11) with 1,5- 
diazidopentane (4). A) A solution of the diacetylene 11 (0.50 g, $1.07 \mathrm{mmol})$ in $\mathrm{CH}_{2} \mathrm{Cl}_{2}(20 \mathrm{~mL})$ and a solution of $\mathrm{CuSO}_{4} \cdot 5 \mathrm{H}_{2} \mathrm{O}$ $(0.11 \mathrm{~g}, 0.43 \mathrm{mmol})$ in water $(0.5 \mathrm{~mL})$, and sodium ascorbate $(0.21 \mathrm{~g}, 1.07 \mathrm{mmol})$ in water $(0.5 \mathrm{~mL})$ were mixed, and the $1,5-$ diazidopentane $4(0.17 \mathrm{~g}, 1.07 \mathrm{mmol})$ was added with stirring at ambient temperature. The temperature was raised to $40^{\circ} \mathrm{C}$ and stirring was continued for $10 \mathrm{~h}$. The cooled mixture was diluted with water $(10 \mathrm{~mL})$, the organic phase was separated, washed with water $(3 \times 50 \mathrm{~mL})$, dried over $\mathrm{MgSO}_{4}$ and filtered. The solvent was evaporated, the residue was subjected to column chromatography on silica gel (eluent chloroform-methanol, 50:1) to isolate $0.058 \mathrm{~g}$ (7\%) of diazide 13, $0.086 \mathrm{~g}$ (13\%) of macroheterocyclic compound 14, $0.279 \mathrm{~g} \mathrm{(42 \% )}$ of cyclic dimer 15 and $0.133 \mathrm{~g} \mathrm{(20 \% )} \mathrm{of} \mathrm{cyclic}$ trimer 16. B) A solution of the diacetylene $11(0.50 \mathrm{~g}, 1.07 \mathrm{mmol})$ in $\mathrm{CH}_{2} \mathrm{Cl}_{2}(107 \mathrm{ml})$ and a solution of $\mathrm{CuSO}_{4} \cdot 5 \mathrm{H}_{2} \mathrm{O}(0.11 \mathrm{~g}, 0.43 \mathrm{mmol})$ in water $(0.5 \mathrm{~mL})$, and sodium ascorbate $(0.21 \mathrm{~g}, 1.07 \mathrm{mmol})$ in water $(0.5 \mathrm{~mL})$ were mixed, and the 1,5-diazidopentane $4(0.17 \mathrm{~g}$, $1.07 \mathrm{mmol}$ ) was added with stirring at ambient temperature. The temperature was raised to $40{ }^{\circ} \mathrm{C}$ and stirring was continued for $90 \mathrm{~h}$. The cooled mixture was diluted with water $(10 \mathrm{~mL})$, the organic phase was separated, washed with water $(3 \times 50 \mathrm{~mL})$, dried over $\mathrm{MgSO}_{4}$, filtered and evaporated. Column chromatography on silica gel (eluent chloroform-methanol, 50:1) gave $0.452 \mathrm{~g} \mathrm{(68 \% )}$ of compound 14 and $0.08 \mathrm{~g}$ (12\%) of compound 15 .

(1S,4aR, 5S,8aR)-Methyl-5-\{2-[2,5-bi\{[(1-(5-azidopentyl)1H-1,2,3-triazol-4-yl]methoxy\}methyl)furan-3-yl]ethyl\}-1,4adimethyl-6-methylenedecahydronaphthalene-1-carboxylate (13), oily substance. $[\alpha]_{\mathrm{D}}+18.45^{\circ}$ (c $1.03 ; \mathrm{CHCl}_{3}$ ). Found: $\mathrm{C} 60.77, \mathrm{H}$ 7.34, N 20.78\%. $\mathrm{C}_{39} \mathrm{H}_{58} \mathrm{~N}_{12} \mathrm{O}_{5}$. requires $\mathrm{C} 60.44, \mathrm{H} 7.54, \mathrm{~N} 21.69 \%$. UV (EtOH) $\lambda_{\max }$ nm (lge): 216 (4.14), 283 (3.33). IR (KBr) $v \mathrm{~cm}^{-1}$ : $1049 \mathrm{~m}, 1093 \mathrm{~m}, 1136 \mathrm{~s}, 1155 \mathrm{~m}, 1229 \mathrm{~m}, 1333 \mathrm{~m}, 1358 \mathrm{~m}, 1379 \mathrm{w}$, 1452 m, 1464 m, 1645 w, 1681 w, 1720 s, 1759 m, 2097 s, 2849 m, 2870 s, 2943 s, 3140 s, 3327 s. ${ }^{1} \mathrm{H}$ NMR $\left(\mathrm{CDCl}_{3}, 298 \mathrm{~K}\right) \delta_{\mathrm{H}} \mathrm{ppm}$ : $7.54\left(1 \mathrm{H}, \mathrm{s}, \mathrm{C}^{5} \mathrm{H}\right)^{*}, 7.52\left(1 \mathrm{H}, \mathrm{s}, \mathrm{C}^{5} \mathrm{H}\right)^{*}, 6.21\left(1 \mathrm{H}, \mathrm{s}, \mathrm{C}^{14} \mathrm{H}\right), 4.85$ $\left(1 \mathrm{H}, \mathrm{s}, \mathrm{C}^{17} \mathrm{H}\right), 4.65\left(2 \mathrm{H}, \mathrm{s}, \mathrm{C}^{4} \mathrm{CH}_{2}\right)^{*}, 4.61\left(2 \mathrm{H}, \mathrm{s}, \mathrm{C}^{4} \mathrm{CH}_{2}\right)^{*}, 4.53$ $\left(1 \mathrm{H}, \mathrm{s}, \mathrm{C}^{17} \mathrm{H}\right), 4.46\left(2 \mathrm{H}, \mathrm{s}, \mathrm{C}^{16} \mathrm{CH}_{2}\right) * 4.42\left(2 \mathrm{H}, \mathrm{s}, \mathrm{C}^{15} \mathrm{CH}_{2}\right)^{*}, 4.32$ $\left(2 \mathrm{H}, \text { d.d, } J=7.9 \mathrm{~Hz}, J=4.7 \mathrm{~Hz}, \mathrm{CH}_{2} \mathrm{~N}^{1}\right)^{* *}, 4.33(2 \mathrm{H}$, d.d, $J=7.9 \mathrm{~Hz}$, $\left.J=4.7 \mathrm{~Hz}, \mathrm{CH}_{2} \mathrm{~N}^{1 "}\right)^{* *}, 3.58\left(3 \mathrm{H}, \mathrm{s}, \mathrm{OCH}_{3}\right), 3.25(4 \mathrm{H}, \mathrm{t}, J=6.8 \mathrm{~Hz}$, $\left.2 \mathrm{CH}_{2} \mathrm{~N}_{3}\right), 2.60\left(1 \mathrm{H}, \mathrm{m}, \mathrm{C}^{12} \mathrm{H}\right), 2.37(1 \mathrm{H}$, d.t, $J=12.8 \mathrm{~Hz}, J=2.7 \mathrm{~Hz}$, $\left.\mathrm{C}^{7} \mathrm{H}\right), 2.21\left(1 \mathrm{H}, \mathrm{m}, \mathrm{C}^{12} \mathrm{H}\right), 2.11\left(1 \mathrm{H}\right.$, d.m, $\left.J=12.8 \mathrm{~Hz}, \mathrm{C}^{3} \mathrm{H}\right), 1.91$ $\left(5 \mathrm{H}, \mathrm{m}, \mathrm{CH}_{2} \mathrm{CH}_{2} \mathrm{~N}^{1}, \mathrm{CH}_{2} \mathrm{CH}_{2} \mathrm{~N}^{1}, \mathrm{C}^{6} \mathrm{H}\right), 1.85(1 \mathrm{H}, \mathrm{d} . \mathrm{d}, J=12.8 \mathrm{~Hz}$, $\left.J=4.3 \mathrm{~Hz}, \mathrm{C}^{7} \mathrm{H}\right), 1.75\left(2 \mathrm{H}, \mathrm{m}, \mathrm{C}^{2} \mathrm{H}, \mathrm{C}^{6} \mathrm{H}\right), 1.67\left(1 \mathrm{H}, \mathrm{m}, \mathrm{C}^{1} \mathrm{H}\right), 1.60$ $\left(5 \mathrm{H}, \mathrm{m}, 2 \mathrm{CH}_{2} \mathrm{CH}_{2} \mathrm{~N}_{3}, \mathrm{C}^{11} \mathrm{H}\right), 1.56\left(1 \mathrm{H}, \mathrm{m}, \mathrm{C}^{9} \mathrm{H}\right), 1.53\left(1 \mathrm{H}, \mathrm{m}, \mathrm{C}^{11} \mathrm{H}\right)$, $1.45\left(1 \mathrm{H}, \mathrm{m}, \mathrm{C}^{2} \mathrm{H}\right), 1.38\left(4 \mathrm{H}, \mathrm{m}, 2 \mathrm{CH}_{2}\right), 1.24(1 \mathrm{H}, \mathrm{d} . \mathrm{d}, J=12.8 \mathrm{~Hz}$, $\left.J=3.2 \mathrm{~Hz}, \mathrm{C}^{5} \mathrm{H}\right), 1.14\left(3 \mathrm{H}, \mathrm{s}, \mathrm{C}^{19} \mathrm{H}_{3}\right), 0.98(1 \mathrm{H}$, d.t, $J=13.6 \mathrm{~Hz}$, $\left.J=4.0 \mathrm{~Hz}, \mathrm{C}^{3} \mathrm{H}\right), 0.95\left(1 \mathrm{H}\right.$, d.t, $\left.J=13.3 \mathrm{~Hz}, J=4.7 \mathrm{~Hz}, \mathrm{C}^{1} \mathrm{H}\right), 0.45$ $\left(3 \mathrm{H}, \mathrm{s}, \mathrm{C}^{20} \mathrm{H}_{3}\right) .{ }^{13} \mathrm{C}$ NMR $\left(\mathrm{CDCl}_{3}, 298 \mathrm{~K}\right) \delta_{\mathrm{C}} \mathrm{ppm}: 177.6\left(\mathrm{C}^{18}\right), 150.7$ $\left(\mathrm{C}^{16}\right), 147.9\left(\mathrm{C}^{8}\right), 147.2\left(\mathrm{C}^{15}\right), 145.0\left(\mathrm{C}^{4}\right)^{*}, 144.9\left(\mathrm{C}^{4}\right)^{*}, 125.5\left(\mathrm{C}^{13}\right)$, $122.5\left(\mathrm{C}^{5}\right)^{*}, 122.4\left(\mathrm{C}^{5}\right)^{*}, 111.6\left(\mathrm{C}^{14}\right), 106.3\left(\mathrm{C}^{17}\right), 64.3\left(\mathrm{CH}_{2} \mathrm{C}^{16}\right)$, $63.5\left(\mathrm{CH}_{2} \mathrm{C}^{4}\right)^{*}, 63.4\left(\mathrm{CH}_{2} \mathrm{C}^{4}\right)^{*}, 62.3\left(\mathrm{CH}_{2} \mathrm{C}^{15}\right), 56.1\left(\mathrm{C}^{5}\right), 55.0$ $\left(\mathrm{C}^{9}\right), 51.1^{2}\left(\mathrm{OCH}_{3}\right), 51.0\left(2 \mathrm{CH}_{2} \mathrm{~N}_{3}\right), 49.9\left(\mathrm{CH}_{2} \mathrm{~N}^{1}, \mathrm{CH}_{2} \mathrm{~N}^{1}\right), 44.2$ $\left(\mathrm{C}^{4}\right), 40.1\left(\mathrm{C}^{10}\right), 38.9\left(\mathrm{C}^{1}\right), 38.6\left(\mathrm{C}^{7}\right), 38.1\left(\mathrm{C}^{3}\right), 29.8\left(\mathrm{CH}_{2} \mathrm{CH}_{2} \mathrm{~N}^{1}\right.$, $\left.\mathrm{CH}_{2} \mathrm{CH}_{2} \mathrm{~N}^{1 "}\right), 28.7\left(\mathrm{C}^{19}\right), 23.7\left(2 \mathrm{CH}_{2} \mathrm{CH}_{2} \mathrm{~N}_{3}\right), 26.2\left(\mathrm{C}^{6}\right), 24.6\left(\mathrm{C}^{11}\right)$, $23.7\left(2 \mathrm{CH}_{2}\right), 23.3\left(\mathrm{C}^{12}\right), 19.9\left(\mathrm{C}^{2}\right), 12.6\left(\mathrm{C}^{20}\right)$.

(1S, 4aR, 5S, 8aR)-Methyl-5-(2-(3,10,25-trioxa-13,14,15, 21,22,23-hexaazatetracyclo[19.2.1.1 $\left.1^{5,8} \cdot 1^{12,15}\right]$ hexacosa-1(24), 5, 7, 12(26),13,22-hexaen-6-yl)ethyl)-1,4a-dimethyl-6-methylenedecahydronaphthalene-1-carboxylate $(14)$, oily substance. $[\alpha]_{\mathrm{D}}+31.25^{\circ}$ (c $0.58 ; \mathrm{CHCl}_{3}$ ). Found: C 65.51, H 7.62, N 12.99\%. [M] 639 . $\mathrm{C}_{34} \mathrm{H}_{48} \mathrm{~N}_{6} \mathrm{O}_{5}$. requires $\mathrm{C} 65.78, \mathrm{H} 7.79, \mathrm{~N} \mathrm{13.54 \% .} \mathrm{[M]} \mathrm{620.} \mathrm{UV}$ (EtOH) $\lambda_{\text {max }} \mathrm{nm}$ (lge): 216 (4.08), 282 (3.38). IR (KBr) $v \mathrm{~cm}^{-1}$ : $754 \mathrm{w}, 775 \mathrm{w}, 822 \mathrm{w}, 891 \mathrm{w}, 986 \mathrm{w}, 1049 \mathrm{~m}, 1067 \mathrm{~m}, 1092 \mathrm{~m}$, $1155 \mathrm{~m}, 1229 \mathrm{~m}, 1333 \mathrm{w}, 1362 \mathrm{w}, 1383 \mathrm{w}, 1449 \mathrm{~m}, 1464 \mathrm{~m}, 1531 \mathrm{w}$, 1547 w, 1654 w, 1720 s, 2870 m, 2945 s, 3080 s, 3140 s, 3400 s. ${ }^{1} \mathrm{H}$ NMR $\left(\mathrm{CDCl}_{3}, 298 \mathrm{~K}\right) \delta_{\mathrm{H}} \mathrm{ppm}: 7.38\left(1 \mathrm{H}, \mathrm{s}, \mathrm{C}^{25} \mathrm{H}\right) *, 7.37(1 \mathrm{H}, \mathrm{s}$, $\left.\mathrm{C}^{24} \mathrm{H}\right)^{*}, 6.23\left(1 \mathrm{H}, \mathrm{s}, \mathrm{C}^{7} \mathrm{H}\right), 4.90\left(1 \mathrm{H}, \mathrm{s}, \mathrm{C}^{11} \mathrm{H}\right), 4.59\left(2 \mathrm{H}, \mathrm{s}, \mathrm{C}^{11} \mathrm{H}_{2}\right)^{*}$, $4.58\left(1 \mathrm{H}, \mathrm{s}, \mathrm{C}^{11^{\prime}} \mathrm{H}\right), 4.54\left(2 \mathrm{H}, \mathrm{s}, \mathrm{C}^{2} \mathrm{H}_{2}\right)^{*}, 4.48\left(2 \mathrm{H}, \mathrm{s}, \mathrm{C}^{9} \mathrm{H}_{2}\right), 4.44$ $\left(2 \mathrm{H}, \mathrm{s}, \mathrm{C}^{4} \mathrm{H}_{2}\right), 4.31\left(4 \mathrm{H}, \mathrm{t}, J=6.0 \mathrm{~Hz}, \mathrm{C}^{16} \mathrm{H}_{2}, \mathrm{C}^{20} \mathrm{H}_{2}\right), 3.59(3 \mathrm{H}, \mathrm{s}$,
$\left.\mathrm{OCH}_{3}\right), 2.53\left(1 \mathrm{H}, \mathrm{m}, \mathrm{C}^{10^{\prime} \mathrm{H}}\right), 2.41\left(1 \mathrm{H}, \mathrm{d} . \mathrm{m}, J=11.5 \mathrm{~Hz}, \mathrm{C}^{7} \mathrm{H}\right), 2.25$ $\left(1 \mathrm{H}, \mathrm{m}, \mathrm{C}^{10^{\prime} \mathrm{H}}\right), 2.13\left(1 \mathrm{H}, \mathrm{d} . \mathrm{m}, J=13.2 \mathrm{~Hz}, \mathrm{C}^{2} \mathrm{H}\right), 1.68-1.98(10 \mathrm{H}$, $\left.\mathrm{m}, \mathrm{C}^{9} \mathrm{H}, \mathrm{C}^{4} \mathrm{H}, \mathrm{C}^{3} \mathrm{H}, \mathrm{C}^{8^{\prime}} \mathrm{H}, \mathrm{C}^{7} \mathrm{H}, \mathrm{C}^{8} \mathrm{H}, \mathrm{C}^{17} \mathrm{H}_{2}, \mathrm{C}^{19} \mathrm{H}_{2}\right), 1.58(1 \mathrm{H}, \mathrm{m}$, $\left.\mathrm{C}^{5^{\prime}} \mathrm{H}\right), 1.57\left(1 \mathrm{H}, \mathrm{m}, \mathrm{C}^{9^{\prime}} \mathrm{H}\right), 1.48\left(1 \mathrm{H}, \mathrm{m}, \mathrm{C}^{3} \mathrm{H}\right), 1.19,1.22(2 \mathrm{H}$, all $\left.\mathrm{m}, \mathrm{C}^{18} \mathrm{H}_{2}\right), 1.26\left(1 \mathrm{H}\right.$, d.d, $\left.J=12.2 \mathrm{~Hz}, J=2.8 \mathrm{~Hz}, \mathrm{C}^{8 \mathrm{a}} \mathrm{H}\right), 1.15(3 \mathrm{H}, \mathrm{s}$, $\left.\mathrm{C}^{13^{\prime}} \mathrm{H}_{3}\right), 0.99\left(1 \mathrm{H}\right.$, d.t, $\left.J=13.3 \mathrm{~Hz}, J=4.0 \mathrm{~Hz}, \mathrm{C}^{2} \mathrm{H}\right), 0.96(1 \mathrm{H}$, d.t. $\left.J=11.0 \mathrm{~Hz}, J=2.4 \mathrm{~Hz}, \mathrm{C}^{4} \mathrm{H}\right), 0.48\left(3 \mathrm{H}, \mathrm{s}, \mathrm{C}^{14} \mathrm{H}_{3}\right) \cdot{ }^{13} \mathrm{C} \mathrm{NMR}\left(\mathrm{CDCl}_{3}\right.$, $298 \mathrm{~K}) \delta_{\mathrm{C}}$ ppm: $177.5\left(\mathrm{C}^{12}\right), 150.4\left(\mathrm{C}^{8}\right), 147.6\left(\mathrm{C}^{6}\right), 146.8\left(\mathrm{C}^{5}\right)$, $144.9\left(\mathrm{C}^{1}\right)^{*}, 144.9\left(\mathrm{C}^{12}\right)^{*}, 125.2\left(\mathrm{C}^{6}\right), 122.5\left(\mathrm{C}^{24}, \mathrm{C}^{25}\right), 111.5\left(\mathrm{C}^{7}\right)$, $106.2\left(\mathrm{C}^{11}\right), 63.7\left(\mathrm{C}^{9}\right), 62.5\left(\mathrm{C}^{11}\right)^{*}, 62.3\left(\mathrm{C}^{2}\right)^{*}, 61.4\left(\mathrm{C}^{4}\right), 55.9\left(\mathrm{C}^{8 \mathrm{a}^{\mathrm{a}}}\right)$, $54.8\left(\mathrm{C}^{5^{\prime}}\right), 50.9\left(\mathrm{OCH}_{3}\right), 49.9\left(\mathrm{C}^{16}, \mathrm{C}^{20}\right), 44.0\left(\mathrm{C}^{1}\right), 39.9\left(\mathrm{C}^{4 \mathrm{a}^{\mathrm{a}}}\right), 38.8$ $\left(\mathrm{C}^{4}\right), 38.4\left(\mathrm{C}^{7}\right), 37.9\left(\mathrm{C}^{2}\right), 28.7\left(\mathrm{C}^{19}, \mathrm{C}^{17}\right), 28.5\left(\mathrm{C}^{13^{\prime}}\right), 26.0\left(\mathrm{C}^{8^{7}}\right)$, $24.4\left(\mathrm{C}^{9}\right), 23.0\left(\mathrm{C}^{18}\right), 23.0\left(\mathrm{C}^{10}\right), 19.7\left(\mathrm{C}^{3}\right), 12.4\left(\mathrm{C}^{14}\right)$.

Dimeric compound 15 , oily substance. $[\alpha]_{\mathrm{D}}+26.41^{\circ}$ (c 0.62 ; $\mathrm{CHCl}_{3}$ ). Found: $\mathrm{C} 65.64, \mathrm{H} 7.48, \mathrm{~N} 12.99 \%$. $[M] 1258 . \mathrm{C}_{68} \mathrm{H}_{96} \mathrm{~N}_{12} \mathrm{O}_{10}$. requires $\mathrm{C} 65.78, \mathrm{H} 7.79, \mathrm{~N} 13.54 \%$. [M] 1240. UV (EtOH) $\lambda_{\max } \mathrm{nm}$ (lge): 216 (4.07), 282 (3.41). IR (KBr) $v \mathrm{~cm}^{-1}: 755 \mathrm{w}, 777 \mathrm{w}, 819 \mathrm{w}$, 891 w, 985 w, 1047 m, 1067 m, 1089 m, 1156 m, 1229 m, 1331 w, 1367 w, 1382 w, 1449 m, 1459 m, 1532 w, 1547 w, 1655 w, 1720 s, $2871 \mathrm{~m}, 2943 \mathrm{~s}, 3081 \mathrm{~s}, 3140 \mathrm{~s}, 3405 \mathrm{~s} .{ }^{1} \mathrm{H}$ NMR $\left(\mathrm{CDCl}_{3}, 298 \mathrm{~K}\right)$ $\delta_{\mathrm{H}}$ ppm: 7.59, $7.59\left(2 \mathrm{H} \text {, all s, } 2 \mathrm{C}^{5} \mathrm{H}\right)^{*}, 7.56,7.57\left(2 \mathrm{H} \text {, all s, } 2 \mathrm{C}^{5} \mathrm{H}\right)^{*}$, $6.20\left(2 \mathrm{H}, \mathrm{s}, 2 \mathrm{C}^{14} \mathrm{H}\right), 4.88\left(2 \mathrm{H}, \mathrm{s}, 2 \mathrm{C}^{17} \mathrm{H}\right), 4.64\left(4 \mathrm{H}, \mathrm{s}, 2 \mathrm{C}^{4 "} \mathrm{CH}_{2}\right)^{*}$, $4.60\left(4 \mathrm{H}, \mathrm{s}, 2 \mathrm{C}^{4} \mathrm{CH}_{2}\right)^{*}, 4.54\left(2 \mathrm{H}, \mathrm{s}, 2 \mathrm{C}^{17} \mathrm{H}\right), 4.47\left(4 \mathrm{H}, \mathrm{s}, 2 \mathrm{C}^{15} \mathrm{CH}_{2}\right) *$, $4.43\left(4 \mathrm{H}, \mathrm{s}, 2 \mathrm{C}^{16} \mathrm{CH}_{2}\right)^{*}, 4.29\left(8 \mathrm{H}, \mathrm{t}, J=7.0 \mathrm{~Hz}, 2 \mathrm{CH}_{2} \mathrm{~N}^{1}, 2 \mathrm{CH}_{2} \mathrm{~N}^{1}\right) *$, $3.59\left(6 \mathrm{H}, \mathrm{s}, 2 \mathrm{OCH}_{3}\right), 2.51\left(2 \mathrm{H}, \mathrm{m}, 2 \mathrm{C}^{12} \mathrm{H}\right), 2.39(2 \mathrm{H}, \mathrm{d} . \mathrm{m}, J=12.4 \mathrm{~Hz}$, $\left.2 \mathrm{C}^{7} \mathrm{H}\right), 2.23\left(2 \mathrm{H}, \mathrm{m}, 2 \mathrm{C}^{12} \mathrm{H}\right), 2.12\left(2 \mathrm{H}\right.$, d.m, $\left.J=13.4 \mathrm{~Hz}, 2 \mathrm{C}^{3} \mathrm{H}\right), 1.94$ $\left(2 \mathrm{H}, \mathrm{m}, 2 \mathrm{C}^{6} \mathrm{H}\right), 1.89\left(8 \mathrm{H}, \mathrm{t}, J=7.0 \mathrm{~Hz}, 2 \mathrm{CH}_{2} \mathrm{CH}_{2} \mathrm{~N}^{1}, 2 \mathrm{CH}_{2} \mathrm{CH}_{2} \mathrm{~N}^{1}\right)$, $1.84\left(2 \mathrm{H}, \mathrm{m}, 2 \mathrm{C}^{7} \mathrm{H}\right), 1.66,1.73,1.77\left(8 \mathrm{H}\right.$, all $\mathrm{m}, 2 \mathrm{C}^{11} \mathrm{H}, 2 \mathrm{C}^{1} \mathrm{H}, 2 \mathrm{C}^{2} \mathrm{H}$, $\left.2 \mathrm{C}^{6} \mathrm{H}\right), 1.56\left(4 \mathrm{H}, \mathrm{m}, 2 \mathrm{C}^{9} \mathrm{H}, 2 \mathrm{C}^{11} \mathrm{H}\right), 1.46\left(2 \mathrm{H}, \mathrm{m}, 2 \mathrm{C}^{2} \mathrm{H}\right), 1.30(4 \mathrm{H}$, $\left.\mathrm{m}, 2 \mathrm{CH}_{2}\right), 1.25\left(2 \mathrm{H}\right.$, d.d, $\left.J=12.9 \mathrm{~Hz}, J=3.0 \mathrm{~Hz}, 2 \mathrm{C}^{5} \mathrm{H}\right), 1.14(6 \mathrm{H}, \mathrm{s}$, $\left.2 \mathrm{C}^{19} \mathrm{H}_{3}\right), 0.99\left(2 \mathrm{H}\right.$, d.t, $\left.J=13.4 \mathrm{~Hz}, J=4.0 \mathrm{~Hz}, 2 \mathrm{C}^{3} \mathrm{H}\right), 0.95(2 \mathrm{H}$, d.t, $\left.J=12.6 \mathrm{~Hz}, J=3.8 \mathrm{~Hz}, 2 \mathrm{C}^{1} \mathrm{H}\right), 0.46\left(6 \mathrm{H}, \mathrm{s}, 2 \mathrm{C}^{20} \mathrm{H}_{3}\right) \cdot{ }^{13} \mathrm{C} \mathrm{NMR}\left(\mathrm{CDCl}_{3}\right.$, $298 \mathrm{~K}) \delta_{\mathrm{C}} \mathrm{ppm}: 177.5\left(2 \mathrm{C}^{18}\right), 150.6\left(2 \mathrm{C}^{15}\right), 147.7\left(2 \mathrm{C}^{8}\right), 146.9\left(2 \mathrm{C}^{16}\right)$, $144.8\left(2 \mathrm{C}^{4}\right)^{*}, 144.6\left(\mathrm{C}^{4^{\prime \prime}}\right)^{*}, 125.3\left(\mathrm{~s}, 2 \mathrm{C}^{13}\right), 122.6\left(2 \mathrm{C}^{5^{\prime \prime}}\right)^{*}, 122.4$ $\left(2 \mathrm{C}^{5}\right)^{*}, 111.5\left(2 \mathrm{C}^{14}\right), 106.2\left(2 \mathrm{C}^{17}\right), 64.2\left(2 \mathrm{CH}_{2} \mathrm{C}^{15}\right), 63.2\left(2 \mathrm{CH}_{2} \mathrm{C}^{4}\right)^{*}$, $63.1\left(2 \mathrm{CH}_{2} \mathrm{C}^{4}\right)^{*}, 62.1\left(2 \mathrm{CH}_{2} \mathrm{C}^{16}\right), 55.9\left(2 \mathrm{C}^{5}\right), 54.8\left(2 \mathrm{C}^{9}\right), 50.9$ $\left(2 \mathrm{OCH}_{3}\right), 49.6\left(2 \mathrm{CH}_{2} \mathrm{~N}^{1}, 2 \mathrm{CH}_{2} \mathrm{~N}^{1 \prime}\right), 44.0\left(2 \mathrm{C}^{4}\right), 39.9\left(2 \mathrm{C}^{10}\right), 38.8$ $\left(2 \mathrm{C}^{1}\right), 38.5\left(2 \mathrm{C}^{7}\right), 37.9\left(2 \mathrm{C}^{3}\right), 29.4\left(6 \mathrm{CH}_{2}\right), 28.6\left(2 \mathrm{C}^{19}\right), 26.0\left(2 \mathrm{C}^{6}\right)$, $24.4\left(2 \mathrm{C}^{11}\right), 23.1\left(2 \mathrm{C}^{12}\right), 19.7\left(2 \mathrm{C}^{2}\right), 12.4\left(2 \mathrm{C}^{20}\right)$.

Trimeric compound 16, oily substance. $[\alpha]_{\mathrm{D}}+26.41^{\circ}$ (c 0.62 ; $\mathrm{CHCl}_{3}$ ). Found: C 65.63, H 7.85, N 13.28\%. [M] 1774. $\mathrm{C}_{102} \mathrm{H}_{144} \mathrm{~N}_{18} \mathrm{O}_{15}$ requires C 65.78, H 7.79, N 13.54\%. [M] 1860. UV (EtOH) $\lambda_{\text {max }} \mathrm{nm}$ (lge): 216 (4.07), 282 (3.41). IR (KBr) $v \mathrm{~cm}^{-1}: 756 \mathrm{w}, 777 \mathrm{w}, 821 \mathrm{w}$, $892 \mathrm{w}, 985$ w, 1049 m, 1066 m, 1089 m, 1155 m, 1228 m, 1332 w, 1366 w, 1382 w, 1449 m, 1459 m, 1533 w, 1548 w, 1654 w, 1720 s, 2871 m, 2942 s, 3082 s, 3139 s, 3405 s. ${ }^{1} \mathrm{H}$ NMR $\left(\mathrm{CDCl}_{3}, 298 \mathrm{~K}\right)$ $\delta_{\mathrm{H}}$ ppm: $7.55\left(3 \mathrm{H}, \mathrm{s}, 3 \mathrm{C}^{5 \prime} \mathrm{H}\right)^{*}, 7.52\left(3 \mathrm{H}, \mathrm{s}, 3 \mathrm{C}^{5} \mathrm{H}\right)^{*}, 6.21(3 \mathrm{H}, \mathrm{s}$, $\left.3 \mathrm{C}^{14} \mathrm{H}\right), 4.85\left(3 \mathrm{H}, \mathrm{s}, 3 \mathrm{C}^{17} \mathrm{H}\right), 4.63\left(6 \mathrm{H}, \mathrm{s}, 3 \mathrm{C}^{4 "} \mathrm{CH}_{2}\right)^{*}, 4.59(6 \mathrm{H}, \mathrm{s}$, $\left.3 \mathrm{C}^{4} \mathrm{CH}_{2}\right)^{*}, 4.52\left(3 \mathrm{H}, \mathrm{s}, 3 \mathrm{C}^{17} \mathrm{H}\right), 4.46\left(6 \mathrm{H}, \mathrm{s}, 3 \mathrm{C}^{15} \mathrm{CH}_{2}\right) *, 4.42(6 \mathrm{H}$, $\left.\mathrm{s}, 3 \mathrm{C}^{16} \mathrm{CH}_{2}\right)^{*}, 4.29\left(12 \mathrm{H}, \mathrm{t}, J=5.6 \mathrm{~Hz}, 3 \mathrm{CH}_{2} \mathrm{~N}^{1}, 3 \mathrm{CH}_{2} \mathrm{~N}^{1 "}\right) *, 3.57$ $\left(9 \mathrm{H}, \mathrm{s}, 3 \mathrm{OCH}_{3}\right), 2.50\left(3 \mathrm{H}, \mathrm{m}, 3 \mathrm{C}^{12} \mathrm{H}\right), 2.37(3 \mathrm{H}, \mathrm{d} . \mathrm{m}, J=11.6 \mathrm{~Hz}$ $\left.3 \mathrm{C}^{7} \mathrm{H}\right), 2.21\left(3 \mathrm{H}, \mathrm{m}, 3 \mathrm{C}^{12} \mathrm{H}\right), 2.11\left(3 \mathrm{H}\right.$, d.m, $\left.J=12.9 \mathrm{~Hz}, 3 \mathrm{C}^{3} \mathrm{H}\right), 1.90$ $\left(15 \mathrm{H}, \mathrm{m}, 3 \mathrm{CH}_{2} \mathrm{CH}_{2} \mathrm{~N}^{1}, 3 \mathrm{CH}_{2} \mathrm{CH}_{2} \mathrm{~N}^{1}, 3 \mathrm{C}^{6} \mathrm{H}\right), 1.81\left(3 \mathrm{H}, \mathrm{m}, 3 \mathrm{C}^{7} \mathrm{H}\right)$, $1.68,1.72,1.75^{2}\left(12 \mathrm{H}\right.$, all $\left.\mathrm{m}, 3 \mathrm{C}^{11} \mathrm{H}, 3 \mathrm{C}^{1} \mathrm{H}, 3 \mathrm{C}^{2} \mathrm{H}, 3 \mathrm{C}^{6} \mathrm{H}\right), 1.55(3 \mathrm{H}$, $\left.\mathrm{m}, 3 \mathrm{C}^{9} \mathrm{H}\right), 1.52\left(3 \mathrm{H}, \mathrm{m}, 3 \mathrm{C}^{11} \mathrm{H}\right), 1.46\left(3 \mathrm{H}, \mathrm{m}, 3 \mathrm{C}^{2} \mathrm{H}\right), 1.32(6 \mathrm{H}, \mathrm{m}$, $\left.3 \mathrm{CH}_{2}\right), 1.24\left(3 \mathrm{H}\right.$, d.d, $\left.J=12.1 \mathrm{~Hz}, J=2.7 \mathrm{~Hz}, 3 \mathrm{C}^{5} \mathrm{H}\right), 1.13(9 \mathrm{H}, \mathrm{s}$, $\left.3 \mathrm{C}^{19} \mathrm{H}_{3}\right), 0.98\left(3 \mathrm{H}\right.$, d.t, $\left.J=13.4 \mathrm{~Hz}, J=3.8 \mathrm{~Hz}, 3 \mathrm{C}^{3} \mathrm{H}\right), 0.95(3 \mathrm{H}$, d.t, $\left.J=13.4 \mathrm{~Hz}, J=3.0 \mathrm{~Hz}, 3 \mathrm{C}^{1} \mathrm{H}\right), 0.45\left(9 \mathrm{H}, \mathrm{s}, 3 \mathrm{C}^{20} \mathrm{H}_{3}\right) \cdot{ }^{13} \mathrm{C} \mathrm{NMR}\left(\mathrm{CDCl}_{3}\right.$, $298 \mathrm{~K}) \delta_{\mathrm{C}}$ ppm: $177.7\left(3 \mathrm{C}^{18}\right), 150.8\left(3 \mathrm{C}^{15}\right), 147.9\left(3 \mathrm{C}^{8}\right), 147.2\left(3 \mathrm{C}^{16}\right)$, $145.0\left(3 \mathrm{C}^{4}\right)^{*}, 144.9\left(3 \mathrm{C}^{4 "}\right) *, 125.5\left(3 \mathrm{C}^{13}\right), 122.2,122.3\left(3 \mathrm{C}^{5^{\prime \prime}}\right) *$, 122.0, $122.1\left(3 \mathrm{C}^{5}\right) *, 111.7\left(3 \mathrm{C}^{14}\right), 106.4\left(3 \mathrm{C}^{17}\right), 64.4\left(3 \mathrm{CH}_{2} \mathrm{C}^{15}\right), 63.4$ $\left(3 \mathrm{CH}_{2} \mathrm{C}^{4 "}\right)^{*}, 63.3\left(3 \mathrm{CH}_{2} \mathrm{C}^{4}\right)^{*}, 62.3\left(3 \mathrm{CH}_{2} \mathrm{C}^{16}\right), 56.1\left(3 \mathrm{C}^{5}\right), 55.0\left(3 \mathrm{C}^{9}\right)$, $51.1\left(3 \mathrm{OCH}_{3}\right), 49.8\left(3 \mathrm{CH}_{2} \mathrm{~N}^{1}, 3 \mathrm{CH}_{2} \mathrm{~N}^{1 "}\right), 44.2\left(3 \mathrm{C}^{4}\right), 40.1\left(3 \mathrm{C}^{10}\right), 39.0$ $\left(3 \mathrm{C}^{1}\right), 38.6\left(3 \mathrm{C}^{7}\right), 38.1\left(3 \mathrm{C}^{3}\right), 29.6\left(9 \mathrm{CH}_{2}\right), 28.7\left(3 \mathrm{C}^{19}\right), 26.2\left(3 \mathrm{C}^{6}\right)$, $24.6\left(3 \mathrm{C}^{11}\right), 23.3\left(3 \mathrm{C}^{12}\right), 19.9\left(3 \mathrm{C}^{2}\right), 12.6\left(3 \mathrm{C}^{20}\right)$.

Reaction of dialkynyl labdatrienoate 11 with 1,10-diazidodecane (5). Diazide $5(0.24 \mathrm{~g}, 1.07 \mathrm{mmol})$, was added to a mixture of a solution of compound $11(0.50 \mathrm{~g}, 1.07 \mathrm{mmol})$ in dichloromethane 
$(107 \mathrm{~mL})$, a solution of $\mathrm{CuSO}_{4} \cdot 5 \mathrm{H}_{2} \mathrm{O}(0.11 \mathrm{~g}, 0.43 \mathrm{mmol})$ in $\mathrm{H}_{2} \mathrm{O}$ $(0.5 \mathrm{~mL})$ and a solution of sodium ascorbate $(0.21 \mathrm{~g}, 1.07 \mathrm{mmol})$ in $\mathrm{H}_{2} \mathrm{O}(0.5 \mathrm{~mL})$ with stirring. The temperature was raised to $40{ }^{\circ} \mathrm{C}$ and stirring was continued for $90 \mathrm{~h}$. The cooled mixture was diluted with water $(10 \mathrm{~mL})$, the organic phase was separated, washed with water $(3 \times 50 \mathrm{~mL})$, and dried over $\mathrm{MgSO}_{4}$. The solvent was evaporated, the residue was subjected to chromatography on silica gel (eluent chloroform-methanol, 100:2) to isolate $0.394 \mathrm{~g} \mathrm{(53 \% )}$ of compound $\mathbf{1 7}$ and $0.220 \mathrm{~g}(30 \%)$ of dimeric compound $\mathbf{1 8}$.

(1S,4aR,5S,8aR)-Methyl-5-(2-\{3,10,30-trioxa-13,14,15,26, 27,28-hexaazatetracyclo[24.2.1.1 $\left.1^{5,8} \cdot 1^{12,15}\right]$ heptriaconta-1(29),5,7, 12(31),13,27-hexaen-6-yl\}ethyl)-1,4a-dimethyl-6-methylenedecahydronaphthalene-1-carboxylate (17), oily substance. Found: $\mathrm{C}$ 67.48, H 8.54, N 11.78\%. [M] 720. $\mathrm{C}_{39} \mathrm{H}_{58} \mathrm{~N}_{6} \mathrm{O}_{5}$. requires C 67.80, $\mathrm{H}$ 8.46, N 12.16\%. [M] 691. UV (EtOH) $\lambda_{\text {max }} \mathrm{nm}$ (lge): 221 (4.09), 285 (3.11). IR (KBr) $v \mathrm{~cm}^{-1}$ : $667 \mathrm{w}, 754 \mathrm{w}, 822 \mathrm{w}, 891 \mathrm{w}, 922 \mathrm{w}, 964 \mathrm{w}$, $1051 \mathrm{~m}, 1072 \mathrm{~m}, 1153 \mathrm{~m}, 1229 \mathrm{~m}, 1333 \mathrm{w}, 1360 \mathrm{w}, 1449 \mathrm{~m}, 1464 \mathrm{~m}$, 1558 m, 1643 w, 1682 w, 1720 s, 2855 m, 2930 m, 3078 s, 3136 s. ${ }^{1} \mathrm{H}$ NMR $\left(\mathrm{CDCl}_{3}, 298 \mathrm{~K}\right) \delta_{\mathrm{H}} \mathrm{ppm}: 7.55\left(1 \mathrm{H}, \mathrm{s}, \mathrm{C}^{30} \mathrm{H}\right) *$, $7.52(1 \mathrm{H}, \mathrm{s}$, $\left.\mathrm{C}^{29} \mathrm{H}\right)^{*}, 6.21\left(1 \mathrm{H}, \mathrm{s}, \mathrm{C}^{7} \mathrm{H}\right), 4.88\left(1 \mathrm{H}, \mathrm{s}, \mathrm{C}^{11^{\prime}} \mathrm{H}\right), 4.58(2 \mathrm{H}, \mathrm{d}, J=1.6 \mathrm{~Hz}$, $\left.\mathrm{C}^{2} \mathrm{H}_{2}\right) *, 4.61\left(2 \mathrm{H}, \mathrm{d}, J=1.6 \mathrm{~Hz}, \mathrm{C}^{11} \mathrm{H}_{2}\right) *, 4.54\left(1 \mathrm{H}, \mathrm{s}, \mathrm{C}^{11} \mathrm{H}\right), 4.46(2 \mathrm{H}$, s, $\left.\mathrm{C}^{9} \mathrm{H}_{2}\right), 4.42\left(2 \mathrm{H}, \mathrm{s}, \mathrm{C}^{4} \mathrm{H}_{2}\right), 4.30\left(4 \mathrm{H}, \mathrm{t}, J=6.4 \mathrm{~Hz}, \mathrm{C}^{16} \mathrm{H}_{2}, \mathrm{C}^{25} \mathrm{H}_{2}, J\right.$ 6.4), $3.57\left(3 \mathrm{H}, \mathrm{s}, \mathrm{OCH}_{3}\right), 2.51\left(1 \mathrm{H}, \mathrm{m}, \mathrm{C}^{10^{\prime} \mathrm{H}}\right), 2.38$ (1H, d.m, $J=11.8$ $\left.\mathrm{Hz}, \mathrm{C}^{7} \mathrm{H}\right), 2.23\left(1 \mathrm{H}, \mathrm{m}, \mathrm{C}^{10^{\prime}} \mathrm{H}\right), 2.11\left(1 \mathrm{H}, \mathrm{d} . \mathrm{m}, J=12.4 \mathrm{~Hz}, \mathrm{C}^{2} \mathrm{H}\right), 1.94$ $\left(1 \mathrm{H}, \mathrm{m}, \mathrm{C}^{8} \mathrm{H}\right), 1.86\left(5 \mathrm{H}, \mathrm{m}, \mathrm{C}^{7} \mathrm{H}, \mathrm{C}^{17} \mathrm{H}_{2}, \mathrm{C}^{24} \mathrm{H}_{2}\right), 1.76\left(1 \mathrm{H}, \mathrm{m}, \mathrm{C}^{8} \mathrm{H}\right)$, $1.73\left(2 \mathrm{H}, \mathrm{m}, \mathrm{C}^{4} \mathrm{H}, \mathrm{C}^{3} \mathrm{H}\right), 1.67\left(1 \mathrm{H}, \mathrm{m}, \mathrm{C}^{9} \mathrm{H}\right), 1.56\left(1 \mathrm{H}, \mathrm{m}, \mathrm{C}^{5^{\prime}} \mathrm{H}\right)$, $1.54\left(1 \mathrm{H}, \mathrm{m}, \mathrm{C}^{9} \mathrm{H}\right), 1.45\left(1 \mathrm{H}, \mathrm{m}, \mathrm{C}^{3} \mathrm{H}\right), 1.20\left(13 \mathrm{H}, \mathrm{s}, \mathrm{C}^{8 \mathrm{a}^{\prime}} \mathrm{H}, \mathrm{C}^{18} \mathrm{H}_{2}\right.$, $\left.\mathrm{C}^{19} \mathrm{H}_{2}, \mathrm{C}^{20} \mathrm{H}_{2}, \mathrm{C}^{21} \mathrm{H}_{2}, \mathrm{C}^{22} \mathrm{H}_{2}, \mathrm{C}^{23} \mathrm{H}_{2}\right), 1.13\left(3 \mathrm{H}, \mathrm{s}, \mathrm{C}^{13} \mathrm{H}_{3}\right), 0.97(2 \mathrm{H}, \mathrm{m}$, $\left.\mathrm{C}^{4} \mathrm{H}, \mathrm{C}^{2} \mathrm{H}\right), 0.46\left(3 \mathrm{H}, \mathrm{s}, \mathrm{C}^{14} \mathrm{H}_{3}\right) .{ }^{13} \mathrm{C} \mathrm{NMR}\left(\mathrm{CDCl}_{3}, 298 \mathrm{~K}\right) \delta_{\mathrm{C}} \mathrm{ppm}$ : $177.6\left(\mathrm{C}^{12^{\prime}}\right), 150.7\left(\mathrm{C}^{8}\right), 147.8\left(\mathrm{C}^{6}\right), 147.2\left(\mathrm{C}^{5}\right), 144.8,144.6\left(\mathrm{C}^{1}\right.$, $\left.\mathrm{C}^{12}\right), 125.4\left(\mathrm{C}^{6}\right), 122.5\left(\mathrm{C}^{29}, \mathrm{C}^{30}\right), 111.6\left(\mathrm{C}^{7}\right), 106.3\left(\mathrm{C}^{11}\right), 64.5\left(\mathrm{C}^{9}\right)$, 63.9, $63.8\left(\mathrm{C}^{2}, \mathrm{C}^{11}\right), 62.3\left(\mathrm{C}^{4}\right), 56.0\left(\mathrm{C}^{8 \mathrm{a}^{9}}\right), 54.9\left(\mathrm{C}^{5}\right), 51.0\left(\mathrm{OCH}_{3}\right)$, $50.2\left(\mathrm{C}^{16}, \mathrm{C}^{25}\right), 44.2\left(\mathrm{C}^{1}\right), 40.1\left(\mathrm{C}^{4 \mathrm{a}^{\prime}}\right), 38.9\left(\mathrm{C}^{4}\right), 38.6\left(\mathrm{C}^{7}\right), 38.0\left(\mathrm{C}^{2}\right)$, $29.8\left(\mathrm{C}^{19}, \mathrm{C}^{22}\right), 28.7\left(\mathrm{C}^{13^{\prime}}\right), 28.3\left(\mathrm{C}^{18}, \mathrm{C}^{23}\right), 28.2\left(\mathrm{C}^{20}, \mathrm{C}^{21}\right), 26.2\left(\mathrm{C}^{8}\right)$, $25.7\left(\mathrm{C}^{17}, \mathrm{C}^{24}\right), 24.5\left(\mathrm{C}^{9}\right), 23.3\left(\mathrm{C}^{10}\right), 19.8\left(\mathrm{C}^{3}\right), 12.5\left(\mathrm{C}^{14^{4}}\right)$.

Dimeric compound 18, oily substance. Found: C 67.91, $\mathrm{H}$ 8.54, N 13.22\%. [M] 1450. $\mathrm{C}_{78} \mathrm{H}_{116} \mathrm{~N}_{12} \mathrm{O}_{10}$. requires C 67.80, $\mathrm{H} 8.46, \mathrm{~N} 12.16 \%$. [M] 1382. UV (EtOH) $\lambda_{\max } \mathrm{nm}(\lg \varepsilon): 220$ (4.33), 285 (3.35). IR (KBr) $v \mathrm{~cm}^{-1}: 665 \mathrm{w}, 756 \mathrm{w}, 820 \mathrm{w}, 891 \mathrm{w}$, $922 \mathrm{w}, 988 \mathrm{w}, 1051 \mathrm{~m}, 1074 \mathrm{~m}, 1092 \mathrm{~m}, 1136 \mathrm{~m}, 1153 \mathrm{~m}, 1229 \mathrm{~m}$, $1333 \mathrm{w}, 1362 \mathrm{w}, 1450 \mathrm{~m}, 1464 \mathrm{~m}, 1549 \mathrm{~m}, 1643 \mathrm{w}, 1682 \mathrm{w}, 1722$ s, 1765 w, 2855 m, 2930 m, 3076 s, 3136 s. ${ }^{1} \mathrm{H}$ NMR $\left(\mathrm{CDCl}_{3}, 298\right.$ K) $\delta_{\mathrm{H}}$ ppm: $7.54\left(2 \mathrm{H}, \mathrm{s}, 2 \mathrm{C}^{5} \mathrm{H}\right) *, 7.51\left(2 \mathrm{H}, \mathrm{s}, 2 \mathrm{C}^{5} \mathrm{H}\right)^{*}, 6.21(2 \mathrm{H}, \mathrm{s}$, $\left.2 \mathrm{C}^{14} \mathrm{H}\right), 4.85\left(2 \mathrm{H}, \mathrm{s}, 2 \mathrm{C}^{17} \mathrm{H}\right), 4.65\left(4 \mathrm{H}, \mathrm{s}, 2 \mathrm{C}^{4} \mathrm{CH}_{2}\right) *, 4.61(4 \mathrm{H}, \mathrm{s}$, $\left.2 \mathrm{C}^{4} \mathrm{CH}_{2}\right) *, 4.53\left(2 \mathrm{H}, \mathrm{s}, 2 \mathrm{C}^{17} \mathrm{H}\right), 4.46\left(4 \mathrm{H}, \mathrm{s}, 2 \mathrm{C}^{15} \mathrm{CH}_{2}\right), 4.42(4 \mathrm{H}, \mathrm{s}$, $\left.2 \mathrm{C}^{16} \mathrm{CH}_{2}\right), 4.30\left(4 \mathrm{H}, \mathrm{t}, J=6.9 \mathrm{~Hz}, 2 \mathrm{CH}_{2} \mathrm{~N}^{1}\right) *, 4.30(4 \mathrm{H}, \mathrm{t}, J=6.9 \mathrm{~Hz}$, $\left.2 \mathrm{CH}_{2} \mathrm{~N}^{2}\right) *, 3.58\left(6 \mathrm{H}, \mathrm{s}, 2 \mathrm{OCH}_{3}\right), 2.50\left(2 \mathrm{H}, \mathrm{m}, 2 \mathrm{C}^{12} \mathrm{H}\right), 2.38(2 \mathrm{H}$, d.m, $\left.J=10.4 \mathrm{~Hz}, 2 \mathrm{C}^{7} \mathrm{H}\right), 2.22\left(2 \mathrm{H}, \mathrm{m}, 2 \mathrm{C}^{12} \mathrm{H}\right), 2.12(2 \mathrm{H}, \mathrm{d} . \mathrm{m}, J=12.9$ $\left.\mathrm{Hz}, 2 \mathrm{C}^{3} \mathrm{H}\right), 1.95\left(2 \mathrm{H}, \mathrm{m}, 2 \mathrm{C}^{6} \mathrm{H}\right), 1.85\left(10 \mathrm{H}, \mathrm{m}, 2 \mathrm{C}^{7} \mathrm{H}, 4 \mathrm{CH}_{2}\right), 1.76$, $1.72\left(6 \mathrm{H}\right.$, all m, $\left.2 \mathrm{C}^{1} \mathrm{H}, 2 \mathrm{C}^{2} \mathrm{H}, 2 \mathrm{C}^{6} \mathrm{H}\right), 1.65\left(2 \mathrm{H}, \mathrm{m}, 2 \mathrm{C}^{11} \mathrm{H}\right), 1.56$ $\left(4 \mathrm{H}, \mathrm{m}, 2 \mathrm{C}^{9} \mathrm{H}, 2 \mathrm{C}^{11} \mathrm{H}\right), 1.46\left(2 \mathrm{H}, \mathrm{m}, 2 \mathrm{C}^{2} \mathrm{H}\right), 1.20\left(26 \mathrm{H}, \mathrm{s}, 12 \mathrm{CH}_{2}\right.$, $\left.2 \mathrm{C}^{5} \mathrm{H}\right), 1.14\left(6 \mathrm{H}, \mathrm{s}, 2 \mathrm{C}^{19} \mathrm{H}_{3}\right), 0.98(2 \mathrm{H}$, d.t, $J=13.2 \mathrm{~Hz}, J=4.0 \mathrm{~Hz}$, $\left.2 \mathrm{C}^{3} \mathrm{H}\right), 0.95\left(2 \mathrm{H}\right.$, d.t, $\left.J=13.4 \mathrm{~Hz}, J=3.6 \mathrm{~Hz}, 2 \mathrm{C}^{1} \mathrm{H}\right), 0.45(6 \mathrm{H}, \mathrm{s}$, $\left.2 \mathrm{C}^{20} \mathrm{H}_{3}\right) \cdot{ }^{13} \mathrm{C}$ NMR $\left(\mathrm{CDCl}_{3}, 298 \mathrm{~K}\right) \delta_{\mathrm{C}} \mathrm{ppm}: 177.7\left(2 \mathrm{C}^{18}\right), 150.7$ $\left(2 \mathrm{C}^{15}\right), 147.8\left(2 \mathrm{C}^{8}\right), 147.1\left(2 \mathrm{C}^{16}\right), 144.9,144.7\left(2 \mathrm{C}^{4}, \mathrm{C}^{4}\right), 125.4$ $\left(2 \mathrm{C}^{13}\right), 122.4,122.3\left(2 \mathrm{C}^{5}, 2 \mathrm{C}^{5}\right), 111.6\left(2 \mathrm{C}^{14}\right), 106.3\left(2 \mathrm{C}^{17}\right), 64.3$ $\left(2 \mathrm{CH}_{2} \mathrm{C}^{15}\right), 63.5,63.3\left(2 \mathrm{CH}_{2} \mathrm{C}^{4}, 2 \mathrm{CH}_{2} \mathrm{C}^{4 \prime}\right), 62.2\left(2 \mathrm{CH}_{2} \mathrm{C}^{16}\right), 56.0$ $\left(2 \mathrm{C}^{5}\right), 54.9\left(2 \mathrm{C}^{9}\right), 51.1\left(2 \mathrm{OCH}_{3}\right), 50.2\left(2 \mathrm{CH}_{2} \mathrm{~N}^{1}, 2 \mathrm{CH}_{2} \mathrm{~N}^{1}\right), 44.2$ $\left(2 \mathrm{C}^{4}\right), 40.1\left(2 \mathrm{C}^{10}\right), 38.9\left(2 \mathrm{C}^{1}\right), 38.6\left(2 \mathrm{C}^{7}\right), 38.0\left(2 \mathrm{C}^{3}\right), 28.8\left(4 \mathrm{CH}_{2}\right)$, $29.2\left(4 \mathrm{CH}_{2}\right), 30.2\left(4 \mathrm{CH}_{2}\right), 28.7\left(2 \mathrm{C}^{19}\right), 26.4\left(4 \mathrm{CH}_{2}\right), 26.2\left(2 \mathrm{C}^{6}\right)$, $24.5\left(2 \mathrm{C}^{11}\right), 23.2\left(2 \mathrm{C}^{12}\right), 19.8\left(2 \mathrm{C}^{2}\right), 12.6\left(2 \mathrm{C}^{20}\right)$.

Reaction of dialkynyl labdatrienoate 11 with 1-azido-2-(2azidoethoxy)ethane (6). 1-azido-2-(2-azidoethoxy)ethane $5(0.17 \mathrm{~g}$, $1.07 \mathrm{mmol})$ was added to a mixture of compound $11(0.50 \mathrm{~g}, 1.07$ $\mathrm{mmol})$ in dichloromethane $(107 \mathrm{~mL})$ and solutions of $\mathrm{CuSO}_{4} \cdot 5 \mathrm{H}_{2} \mathrm{O}$ $(0.11 \mathrm{~g}, 0.43 \mathrm{mmol})$ in $\mathrm{H}_{2} \mathrm{O}(0.5 \mathrm{~mL})$ and sodium ascorbate $(0.21 \mathrm{~g}$, $1.07 \mathrm{mmol})$ in $\mathrm{H}_{2} \mathrm{O}(0.5 \mathrm{~mL})$ with stirring. The temperature was raised to $40{ }^{\circ} \mathrm{C}$ and stirring was continued for $90 \mathrm{~h}$. The cooled mixture was diluted with water $(10 \mathrm{~mL})$, the organic phase was separated, washed with water $(3 \times 50 \mathrm{~mL})$, dried over $\mathrm{MgSO}_{4}$ and filtered. The solvent was evaporated, the residue was subjected to chromatography on silica gel (eluent chloroform-methanol, 50:1) to isolate $0.255 \mathrm{~g} \mathrm{(38 \% )}$ of compound 19, $0.154 \mathrm{~g} \mathrm{(23 \% )} \mathrm{of} \mathrm{dimeric}$ compound 20 and $0.174 \mathrm{~g}(26 \%)$ of trimeric compound 21 .

(1S,4aR, 5S, 8aR)-Methyl-5-(2-\{3,10,18,25-tetraoxa-13,14, $15,21,22,23$-hexaazatetracyclo[19.2.1.1 $\left.1^{5,8} \cdot 1^{12,15}\right]$ hexacosa-1(24), 5,7,12(26),13,22-hexaen-6-yl\}ethyl)-1,4a-dimethyl-6-methylenedecahydronaphthalene-1-carboxylate (19), oily substance. $[\alpha]_{D}+14.85^{\circ}(\mathrm{c}$ 3.07; $\mathrm{CHCl}_{3}$ ). Found: $\mathrm{C} 63.95, \mathrm{H} 7.67, \mathrm{~N} 12.98 \%$. [M] 624. $\mathrm{C}_{33} \mathrm{H}_{46} \mathrm{~N}_{6} \mathrm{O}_{6}$. requires $\mathrm{C}$ 63.65, H 7.45, N 13.49\%. [M] 622. UV (EtOH) $\lambda_{\text {max }} \mathrm{nm}$ (lge): 221 (4.04), 280 (2.61). IR (KBr) $v \mathrm{~cm}^{-1}: 754 \mathrm{w}, 820 \mathrm{w}, 891 \mathrm{w}$, $955 \mathrm{w}, 988 \mathrm{w}, 1049 \mathrm{~m}, 1072 \mathrm{~m}, 1109 \mathrm{~m}, 1134 \mathrm{~m}, 1153 \mathrm{~m}, 1229 \mathrm{~m}$, $1333 \mathrm{w}, 1360 \mathrm{w}, 1450 \mathrm{~m}, 1464 \mathrm{~m}, 1558 \mathrm{w}, 1643 \mathrm{w}, 1720 \mathrm{~s}, 2855 \mathrm{~m}$, $2870 \mathrm{~m}, 2945 \mathrm{~s}, 3078 \mathrm{~s}, 3142 \mathrm{s.}{ }^{1} \mathrm{H}$ NMR $\left(\mathrm{CDCl}_{3}, 298 \mathrm{~K}\right) \delta_{\mathrm{H}} \mathrm{ppm}: 7.47$ $\left(1 \mathrm{H}, \mathrm{s}, \mathrm{C}^{25} \mathrm{H}\right)^{*}, 7.46\left(1 \mathrm{H}, \mathrm{s}, \mathrm{C}^{24} \mathrm{H}\right)^{*}, 6.23\left(1 \mathrm{H}, \mathrm{s}, \mathrm{C}^{7} \mathrm{H}\right), 4.89(1 \mathrm{H}, \mathrm{s}$, $\left.\mathrm{C}^{11^{\prime}} \mathrm{H}\right), 4.58\left(2 \mathrm{H}, \mathrm{s}, \mathrm{C}^{11} \mathrm{H}_{2}\right)^{*}, 4.55\left(1 \mathrm{H}, \mathrm{s}, \mathrm{C}^{11^{\prime}} \mathrm{H}\right), 4.51\left(2 \mathrm{H}, \mathrm{s}, \mathrm{C}^{2} \mathrm{H}_{2}\right)^{*}$, $4.49\left(2 \mathrm{H}, \mathrm{d}, J=3.1 \mathrm{~Hz}, \mathrm{C}^{9} \mathrm{H}_{2}\right), 4.47\left(4 \mathrm{H}, \mathrm{m}, \mathrm{C}^{16} \mathrm{H}_{2}, \mathrm{C}^{20} \mathrm{H}_{2}\right), 4.44(2 \mathrm{H}, \mathrm{s}$, $\left.\mathrm{C}^{4} \mathrm{H}_{2}\right), 3.78\left(4 \mathrm{H}, \mathrm{q}, J=4.7 \mathrm{~Hz}, \mathrm{C}^{17} \mathrm{H}_{2}, \mathrm{C}^{19} \mathrm{H}_{2}\right), 3.57\left(3 \mathrm{H}, \mathrm{s}, \mathrm{OCH}_{3}\right), 2.52$ $\left(1 \mathrm{H}, \mathrm{m}, \mathrm{C}^{10^{\prime} \mathrm{H}}\right), 2.39\left(1 \mathrm{H}, \mathrm{t} . \mathrm{d}, J=12.8 \mathrm{~Hz}, J=3.2 \mathrm{~Hz}, \mathrm{C}^{7} \mathrm{H}\right), 2.24(1 \mathrm{H}$,

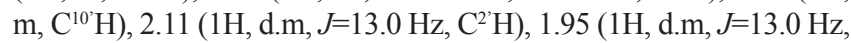
$\left.\mathrm{C}^{8^{\prime} \mathrm{H}}\right), 1.86\left(1 \mathrm{H}\right.$, d.t, $\left.J=12.8 \mathrm{~Hz}, J=4.7 \mathrm{~Hz}, \mathrm{C}^{7} \mathrm{H}\right), 1.76\left(1 \mathrm{H}, \mathrm{m}, \mathrm{C}^{8} \mathrm{H}\right)$, $1.74\left(2 \mathrm{H}, \mathrm{m}, \mathrm{C}^{4} \mathrm{H}, \mathrm{C}^{3} \mathrm{H}\right), 1.67\left(1 \mathrm{H}, \mathrm{m}, \mathrm{C}^{9} \mathrm{H}\right), 1.57\left(1 \mathrm{H}, \mathrm{m}, \mathrm{C}^{5} \mathrm{H}\right), 1.54$ $\left(1 \mathrm{H}, \mathrm{m}, \mathrm{C}^{9} \mathrm{H}\right), 1.46\left(1 \mathrm{H}, \mathrm{m}, \mathrm{C}^{3} \mathrm{H}\right), 1.24(1 \mathrm{H}, \mathrm{d} . \mathrm{d}, J=12.7 \mathrm{~Hz}, J=3.2$ $\left.\mathrm{Hz}, \mathrm{C}^{8 \mathrm{a}} \mathrm{H}\right), 1.13\left(3 \mathrm{H}, \mathrm{s}, \mathrm{C}^{13^{3}} \mathrm{H}_{3}\right), 0.98(1 \mathrm{H}$, d.t, $J=13.0 \mathrm{~Hz}, J=3.8 \mathrm{~Hz}$, $\left.\mathrm{C}^{2} \mathrm{H}\right), 0.95\left(1 \mathrm{H}\right.$, d.t. $\left.J=13.0 \mathrm{~Hz}, J=3.0 \mathrm{~Hz}, \mathrm{C}^{4} \mathrm{H}\right), 0.46\left(3 \mathrm{H}, \mathrm{s}, \mathrm{C}^{14} \mathrm{H}_{3}\right)$. ${ }^{13} \mathrm{C} \mathrm{NMR}\left(\mathrm{CDCl}_{3}, 298 \mathrm{~K}\right) \delta_{\mathrm{C}} \mathrm{ppm}: 177.6\left(\mathrm{C}^{12}\right), 150.7\left(\mathrm{C}^{8}\right), 147.7\left(\mathrm{C}^{6}\right)$, $147.2\left(\mathrm{C}^{5}\right), 144.9\left(\mathrm{C}^{12}, \mathrm{C}^{1}\right), 125.4\left(\mathrm{C}^{6}\right), 123.5\left(\mathrm{C}^{24}\right)^{*}, 123.6\left(\mathrm{C}^{25}\right)^{*}, 111.8$ $\left(\mathrm{C}^{7}\right), 106.4\left(\mathrm{C}^{11}\right), 69.5\left(\mathrm{C}^{19}, \mathrm{C}^{17}\right), 63.8\left(\mathrm{C}^{9}\right), 62.6\left(\mathrm{C}^{11}\right)^{*}, 62.3\left(\mathrm{C}^{2}\right)^{*}, 61.4$ $\left(\mathrm{C}^{4}\right), 56.1\left(\mathrm{C}^{8 \mathrm{a}^{\prime}}\right), 54.9\left(\mathrm{C}^{5}\right), 51.1\left(\mathrm{OCH}_{3}\right), 50.3\left(\mathrm{C}^{16}, \mathrm{C}^{20}\right), 44.2\left(\mathrm{C}^{1}\right)$, $40.1\left(\mathrm{C}^{4 \mathrm{a}^{\prime}}\right), 38.9\left(\mathrm{C}^{4^{\prime}}\right), 38.6\left(\mathrm{C}^{7^{\prime}}\right), 38.1\left(\mathrm{C}^{2^{\prime}}\right), 28.7\left(\mathrm{C}^{13^{\prime}}\right), 26.2\left(\mathrm{C}^{8^{\prime}}\right), 24.6$ $\left(\mathrm{C}^{9^{\prime}}\right), 23.2\left(\mathrm{C}^{10^{\prime}}\right), 19.8\left(\mathrm{C}^{3}\right), 12.6\left(\mathrm{C}^{14^{\prime}}\right)$.

Dimeric compound 20 , oily substance. $[\alpha]_{\mathrm{D}}+30.53^{\circ}$ (c 0.57 ; $\mathrm{CHCl}_{3}$ ). Found: $\mathrm{C}$ 63.81, H 7.67, N 13.58\%. [M] 1302. $\mathrm{C}_{66} \mathrm{H}_{92} \mathrm{~N}_{12} \mathrm{O}_{12}$. requires $\mathrm{C} 63.65, \mathrm{H} 7.45, \mathrm{~N} 13.49 \%$. [M] 1244. UV (EtOH) $\lambda_{\max } \mathrm{nm}$ (lge): 222 (4.45), 280 (3.31). IR (KBr) $v \mathrm{~cm}^{-1}: 754 \mathrm{w}, 818 \mathrm{w}, 891 \mathrm{w}$, 920 w, 988 w, 1049 m, 1069 m, 1134 m, 1151 m, 1227 m, 1333 w, 1360 w, 1449 m, 1462 m, 1547 m, 1558 w, 1643 w, 1720 s, 1763 w, $2855 \mathrm{~m}, 2870 \mathrm{~m}, 2932 \mathrm{~m}, 2945 \mathrm{~s}, 3078 \mathrm{~s}, 3144 \mathrm{~s} .{ }^{1} \mathrm{H} \mathrm{NMR}\left(\mathrm{CDCl}_{3}\right.$, $298 \mathrm{~K}) \delta_{\mathrm{H}}$ ppm: $7.42\left(2 \mathrm{H}, \mathrm{s}, 2 \mathrm{C}^{5 ”} \mathrm{H}\right)^{*}, 7.41,7.40\left(2 \mathrm{H} \text {, all s, 2C }{ }^{5} \mathrm{H}\right)^{*}$, $6.18\left(2 \mathrm{H}, \mathrm{s}, 2 \mathrm{C}^{14} \mathrm{H}\right), 4.85\left(2 \mathrm{H}, \mathrm{s}, 2 \mathrm{C}^{17} \mathrm{H}\right), 4.58(4 \mathrm{H}, \mathrm{d}, J=5.4 \mathrm{~Hz}$, $\left.2 \mathrm{C}^{4 \prime} \mathrm{CH}_{2}\right)^{*}, 4.56\left(4 \mathrm{H}, \mathrm{d}, J=3.8 \mathrm{~Hz}, 2 \mathrm{C}^{4} \mathrm{CH}_{2}\right)^{*}, 4.51\left(2 \mathrm{H}, \mathrm{s}, 2 \mathrm{C}^{17} \mathrm{H}\right)$, $4.43\left(4 \mathrm{H}, \mathrm{d}, J=3.2 \mathrm{~Hz}, 2 \mathrm{C}^{15} \mathrm{CH}_{2}\right)^{*}, 4.40\left(8 \mathrm{H}, \mathrm{m}, 2 \mathrm{CH}_{2} \mathrm{~N}^{1}, 2 \mathrm{CH}_{2} \mathrm{~N}^{1}\right)$, $4.39\left(4 \mathrm{H}, \mathrm{s}, 2 \mathrm{C}^{16} \mathrm{CH}_{2}\right), 3.73\left(8 \mathrm{H}, \mathrm{t}, J=4.6 \mathrm{~Hz}, 4 \mathrm{CH}_{2} \mathrm{O}\right), 3.57(6 \mathrm{H}, \mathrm{s}$, $\left.2 \mathrm{OCH}_{3}\right), 2.48\left(2 \mathrm{H}, \mathrm{m}, 2 \mathrm{C}^{12} \mathrm{H}\right), 2.37\left(2 \mathrm{H}, \mathrm{d} . \mathrm{m}, J=11.8 \mathrm{~Hz}, 2 \mathrm{C}^{7} \mathrm{H}\right)$, $2.19\left(2 \mathrm{H}, \mathrm{m}, 2 \mathrm{C}^{12} \mathrm{H}\right), 2.10\left(2 \mathrm{H}, \mathrm{d} . \mathrm{m}, J=13.4 \mathrm{~Hz}, 2 \mathrm{C}^{3} \mathrm{H}\right), 1.95(2 \mathrm{H}$, d.m, $\left.J=12.0 \mathrm{~Hz}, 2 \mathrm{C}^{6} \mathrm{H}\right), 1.81\left(2 \mathrm{H}\right.$, d.t. $\left.J=14.0 \mathrm{~Hz}, J=3.2 \mathrm{~Hz}, 2 \mathrm{C}^{7} \mathrm{H}\right)$, $1.71,1.75\left(6 \mathrm{H}\right.$, all m, $\left.2 \mathrm{C}^{1} \mathrm{H}, 2 \mathrm{C}^{2} \mathrm{H}, 2 \mathrm{C}^{6} \mathrm{H}\right), 1.63\left(2 \mathrm{H}, \mathrm{m}, 2 \mathrm{C}^{11} \mathrm{H}\right), 1.54$ $\left(4 \mathrm{H}, \mathrm{m}, 2 \mathrm{C}^{9} \mathrm{H}, 2 \mathrm{C}^{11} \mathrm{H}\right), 1.46\left(2 \mathrm{H}, \mathrm{m}, 2 \mathrm{C}^{2} \mathrm{H}\right), 1.23(2 \mathrm{H}$, d.d, $J=12.4$ $\left.\mathrm{Hz}, J=2.7 \mathrm{~Hz}, 2 \mathrm{C}^{5} \mathrm{H}\right), 1.13\left(6 \mathrm{H}, \mathrm{s}, 2 \mathrm{C}^{19} \mathrm{H}_{3}\right), 0.97(2 \mathrm{H}$, d.t, $J=13.4$ $\left.\mathrm{Hz}, J=4.3 \mathrm{~Hz}, 2 \mathrm{C}^{3} \mathrm{H}\right), 0.94$ (2H, d.t, $\left.J=13.4 \mathrm{~Hz}, J=3.8 \mathrm{~Hz}, 2 \mathrm{C}^{1} \mathrm{H}\right)$, $0.44\left(6 \mathrm{H}, \mathrm{s}, 2 \mathrm{C}^{20} \mathrm{H}_{3}\right) \cdot{ }^{13} \mathrm{C} \mathrm{NMR}\left(\mathrm{CDCl}_{3}, 298 \mathrm{~K}\right) \delta_{\mathrm{C}} \mathrm{ppm:} 177.6\left(2 \mathrm{C}^{18}\right)$, 150.70, $150.72\left(2 \mathrm{C}^{15}\right), 147.7\left(2 \mathrm{C}^{8}\right), 147.08,147.11\left(2 \mathrm{C}^{16}\right), 144.7$ $\left(2 \mathrm{C}^{4}\right)^{*}, 144.56,144.59\left(\mathrm{C}^{4^{\prime \prime}}\right) *, 125.29,125.32\left(2 \mathrm{C}^{13}\right), 123.90,123.93$ $\left(2 \mathrm{C}^{5}\right)^{*}, 123.80,123.83\left(2 \mathrm{C}^{5}\right) *, 111.53,111.56\left(2 \mathrm{C}^{14}\right), 106.3\left(2 \mathrm{C}^{17}\right)$, $69.10,69.12\left(2 \mathrm{CH}_{2} \mathrm{O}\right), 69.1\left(2 \mathrm{CH}_{2} \mathrm{O}\right), 64.26,64.30\left(2 \mathrm{CH}_{2} \mathrm{C}^{15}\right), 63.3$ $\left(2 \mathrm{CH}_{2} \mathrm{C}^{4}\right)^{*}, 63.2\left(2 \mathrm{CH}_{2} \mathrm{C}^{4}\right) *, 62.1\left(2 \mathrm{CH}_{2} \mathrm{C}^{16}\right), 55.9\left(2 \mathrm{C}^{5}\right), 54.9\left(2 \mathrm{C}^{9}\right)$, $51.1\left(2 \mathrm{OCH}_{3}\right), 49.9\left(2 \mathrm{CH}_{2} \mathrm{~N}^{1}, 2 \mathrm{CH}_{2} \mathrm{~N}^{1}\right), 44.1\left(2 \mathrm{C}^{4}\right), 40.1\left(2 \mathrm{C}^{10}\right), 38.9$ $\left(2 \mathrm{C}^{1}\right), 38.6\left(2 \mathrm{C}^{7}\right), 37.9\left(2 \mathrm{C}^{3}\right), 28.7\left(2 \mathrm{C}^{19}\right), 26.1\left(2 \mathrm{C}^{6}\right), 24.5\left(2 \mathrm{C}^{11}\right)$, $23.2\left(2 \mathrm{C}^{12}\right), 19.8\left(2 \mathrm{C}^{2}\right), 12.5\left(2 \mathrm{C}^{20}\right)$.

Trimeric compound 21, oily substance. $[\alpha]_{D}+25.48^{\circ}$ (c 2.74; $\mathrm{CHCl}_{3}$ ). Found: C 63.48, H 7.75, N 13.66\%. [M] 1788. $\mathrm{C}_{99} \mathrm{H}_{138} \mathrm{~N}_{18} \mathrm{O}_{18}$. requires C 63.65, H 7.45, N 13.49\%. [M] 1866. UV (EtOH) $\lambda_{\max } \mathrm{nm}(\lg \varepsilon)$ : 221 (4.44), 280 (3.35). IR (KBr) $v \mathrm{~cm}^{-1}: 756 \mathrm{w}, 820 \mathrm{w}, 893 \mathrm{w}, 922 \mathrm{w}$, $988 \mathrm{w}, 1051 \mathrm{~m}, 1069 \mathrm{~m}, 1134 \mathrm{~m}, 1151 \mathrm{~m}, 1229 \mathrm{~m}, 1335 \mathrm{w}, 1360 \mathrm{w}$, $1450 \mathrm{~m}, 1464 \mathrm{~m}, 1643 \mathrm{w}, 1664 \mathrm{w}, 1720 \mathrm{~s}, 1761 \mathrm{w}, 2856 \mathrm{~m}, 2870 \mathrm{~m}$, $2930 \mathrm{~m}, 2947 \mathrm{~s}, 3078 \mathrm{~s}, 3142 \mathrm{s.}{ }^{1} \mathrm{H} \mathrm{NMR}\left(\mathrm{CDCl}_{3}, 298 \mathrm{~K}\right) \delta_{\mathrm{H}} \mathrm{ppm}: 7.53$ 
$\left(6 \mathrm{H}, \mathrm{s}, 3 \mathrm{C}^{5} \mathrm{H}, 3 \mathrm{C}^{5} \mathrm{H}\right), 6.19\left(3 \mathrm{H}, \mathrm{s}, 3 \mathrm{C}^{14} \mathrm{H}\right), 4.83\left(3 \mathrm{H}, \mathrm{s}, 3 \mathrm{C}^{17} \mathrm{H}\right), 4.57$ $\left(6 \mathrm{H}, \mathrm{d}, J=3.8 \mathrm{~Hz}, 3 \mathrm{C}^{4 "} \mathrm{CH}_{2}\right)^{*}, 4.55\left(6 \mathrm{H}, \mathrm{d}, J=3.8 \mathrm{~Hz}, 3 \mathrm{C}^{4} \mathrm{CH}_{2}\right)^{*}, 4.50$ $\left(3 \mathrm{H}, \mathrm{s}, 3 \mathrm{C}^{17} \mathrm{H}\right), 4.42\left(18 \mathrm{H}, \mathrm{s}, 3 \mathrm{CH}_{2} \mathrm{~N}^{1}, 3 \mathrm{CH}_{2} \mathrm{~N}^{1}, 3 \mathrm{CH}_{2} \mathrm{C}^{15}\right), 4.39(6 \mathrm{H}$, $\left.\mathrm{s}, 3 \mathrm{C}^{16} \mathrm{CH}_{2}\right), 3.74\left(12 \mathrm{H}, \mathrm{s}, 6 \mathrm{CH}_{2} \mathrm{O}\right), 3.55\left(9 \mathrm{H}, \mathrm{s}, 3 \mathrm{OCH}_{3}\right), 2.47(3 \mathrm{H}, \mathrm{m}$, $\left.3 \mathrm{C}^{12} \mathrm{H}\right), 2.35$ (3H, d.m, $\left.J=11.3 \mathrm{~Hz}, 3 \mathrm{C}^{7} \mathrm{H}\right), 2.19\left(3 \mathrm{H}, \mathrm{m}, 3 \mathrm{C}^{12} \mathrm{H}\right), 2.09$ ( $3 \mathrm{H}$, d.m, $\left.J=12.9 \mathrm{~Hz}, 3 \mathrm{C}^{3} \mathrm{H}\right), 1.93\left(3 \mathrm{H}\right.$, d.m, $\left.J=11.8 \mathrm{~Hz}, 3 \mathrm{C}^{6} \mathrm{H}\right), 1.81$ ( $3 \mathrm{H}$, d.t, $\left.J=14.5 \mathrm{~Hz}, J=3.2 \mathrm{~Hz}, 3 \mathrm{C}^{7} \mathrm{H}\right), 1.70,1.73\left(9 \mathrm{H}\right.$, all m, $3 \mathrm{C}^{1} \mathrm{H}$, $\left.3 \mathrm{C}^{2} \mathrm{H}, 3 \mathrm{C}^{6} \mathrm{H}\right), 1.63\left(3 \mathrm{H}, \mathrm{m}, 3 \mathrm{C}^{11} \mathrm{H}\right), 1.53\left(6 \mathrm{H}, \mathrm{m}, 3 \mathrm{C}^{11} \mathrm{H}, 3 \mathrm{C}^{9} \mathrm{H}\right), 1.44$ $\left(3 \mathrm{H}, \mathrm{m}, 3 \mathrm{C}^{2} \mathrm{H}\right), 1.23\left(3 \mathrm{H}\right.$, d.d, $\left.J=12.4 \mathrm{~Hz}, J=2.7 \mathrm{~Hz}, 3 \mathrm{C}^{5} \mathrm{H}\right), 1.12(9 \mathrm{H}$, s, $\left.3 \mathrm{C}^{19} \mathrm{H}_{3}\right), 0.96\left(3 \mathrm{H}\right.$, d.t, $\left.J=12.9 \mathrm{~Hz}, J=3.2 \mathrm{~Hz}, 3 \mathrm{C}^{3} \mathrm{H}\right), 0.94(3 \mathrm{H}$, d.t, $\left.J=11.8 \mathrm{~Hz}, J=3.8 \mathrm{~Hz}, 3 \mathrm{C}^{1} \mathrm{H}\right), 0.43\left(9 \mathrm{H}, \mathrm{s}, 3 \mathrm{C}^{20} \mathrm{H}_{3}\right) .{ }^{13} \mathrm{C} \mathrm{NMR}\left(\mathrm{CDCl}_{3}\right.$, $298 \mathrm{~K}) \delta_{\mathrm{C}} \mathrm{ppm}: 177.6\left(3 \mathrm{C}^{18}\right), 150.6\left(3 \mathrm{C}^{15}\right), 147.8\left(3 \mathrm{C}^{8}\right), 147.0\left(3 \mathrm{C}^{16}\right)$, 144.71, 144.72, $144.6\left(3 \mathrm{C}^{4}, 3 \mathrm{C}^{4 \prime}\right), 125.4\left(3 \mathrm{C}^{13}\right), 123.7,123.8,123.56$, $123.59\left(3 \mathrm{C}^{5}, 3 \mathrm{C}^{5}\right), 111.6\left(3 \mathrm{C}^{14}\right), 106.3\left(3 \mathrm{C}^{17}\right), 69.2\left(6 \mathrm{CH}_{2} \mathrm{O}\right), 64.2$ $\left(3 \mathrm{CH}_{2} \mathrm{C}^{15}\right), 63.2,63.1\left(3 \mathrm{CH}_{2} \mathrm{C}^{4}, 3 \mathrm{CH}_{2} \mathrm{C}^{4}\right), 62.1\left(3 \mathrm{CH}_{2} \mathrm{C}^{16}\right), 55.9\left(3 \mathrm{C}^{5}\right)$, $54.9\left(3 \mathrm{C}^{9}\right), 51.0\left(3 \mathrm{OCH}_{3}\right), 49.8\left(3 \mathrm{CH}_{2} \mathrm{~N}^{1}, 3 \mathrm{CH}_{2} \mathrm{~N}^{1}\right), 44.1\left(3 \mathrm{C}^{4}\right), 40.0$ $\left(3 \mathrm{C}^{10}\right), 38.9\left(3 \mathrm{C}^{1}\right), 38.6\left(3 \mathrm{C}^{7}\right), 37.9\left(3 \mathrm{C}^{3}\right), 28.6\left(3 \mathrm{C}^{19}\right), 26.1\left(3 \mathrm{C}^{6}\right), 24.5$ $\left(3 \mathrm{C}^{11}\right), 23.2\left(3 \mathrm{C}^{12}\right), 19.8\left(3 \mathrm{C}^{2}\right), 12.5\left(3 \mathrm{C}^{20}\right)$.

Reaction of compound 11 with 1,2-bis(2-azidoethoxy)ethane (7). Diazide $7(0.21 \mathrm{~g}, 1.07 \mathrm{mmol})$, was added to a mixture of a solution of compound $11(0.50 \mathrm{~g}, 1.07 \mathrm{mmol})$ in dichloromethane $(107 \mathrm{~mL})$, a solution of $\mathrm{CuSO}_{4} \cdot 5 \mathrm{H}_{2} \mathrm{O}(0.11 \mathrm{~g}, 0.43 \mathrm{mmol})$ in $\mathrm{H}_{2} \mathrm{O}$ $(0.5 \mathrm{~mL})$ and a solution of sodium ascorbate $(0.21 \mathrm{~g}, 1.07 \mathrm{mmol})$ in $\mathrm{H}_{2} \mathrm{O}(0.5 \mathrm{~mL})$ with stirring. The temperature was raised to $40{ }^{\circ} \mathrm{C}$ and stirring was continued for $90 \mathrm{~h}$. The cooled mixture was diluted with water $(10 \mathrm{~mL})$, the organic phase was separated, washed with water $(3 \times 50 \mathrm{~mL})$, dried over $\mathrm{MgSO}_{4}$ and filtered. The solvent was evaporated, the residue was subjected to column chromatography on silica gel (eluent chloroform-methanol, 50:1) to isolate $0.229 \mathrm{~g}$ (32\%) of compound $\mathbf{2 2}, 0.071 \mathrm{~g}(10 \%)$ of dimeric compound $\mathbf{2 3}$, and $0.221 \mathrm{~g} \mathrm{(31 \% )}$ of trimeric compound 24.

$(1 S, 4 a R, 5 S, 8 a R)-M e t h y l-5-(2-\{3,10,18,21,28-$ pentaoxa$13,14,15,24,25,26$-hexaazatetracyclo-[22.2.1.1 $\left.1^{5,8} \cdot 1^{12,15}\right]$ nonacosa1(27), 5, 7,12(29), 13,25-hexaen-6-yl\}ethyl)-1,4a-dimethyl-6methylene decahydronaphthalene-1-carboxylate (22), oily substance. Found: $\mathrm{C}$ 63.32, H 7.88, N 13.02\%. [M] 654. $\mathrm{C}_{35} \mathrm{H}_{50} \mathrm{~N}_{6} \mathrm{O}_{7}$. requires C 63.04, H 7.56, N 12.60. [M] 666. UV (EtOH) $\lambda_{\text {max }}^{50} \mathrm{~nm}(\lg \varepsilon): 221$ (4.06), 285 (2.75). IR (KBr) $v \mathrm{~cm}^{-1}: 665 \mathrm{w}, 754 \mathrm{w}, 822 \mathrm{w}, 893 \mathrm{w}$, $922 \mathrm{w}, 988 \mathrm{w}, 1051 \mathrm{~m}, 1072 \mathrm{~m}, 1136 \mathrm{~m}, 1150 \mathrm{~m}, 1227 \mathrm{~m}, 1333 \mathrm{w}$, 1358 w, 1450 m, 1464 m, 1553 m, 1643 w, 1682 w, 1720 s, 2870 m, $2945 \mathrm{~m}, 3080 \mathrm{~s}, 3142 \mathrm{s.}{ }^{1} \mathrm{H}$ NMR $\left(\mathrm{CDCl}_{3}, 298 \mathrm{~K}\right) \delta_{\mathrm{H}} \mathrm{ppm}: 7.67,7.66$ (both s, $1 \mathrm{H}, \mathrm{C}^{28} \mathrm{H}$ and $\left.\mathrm{C}^{27} \mathrm{H}\right), 6.04\left(1 \mathrm{H}, \mathrm{s}, \mathrm{C}^{7} \mathrm{H}\right), 4.87\left(1 \mathrm{H}, \mathrm{s}, \mathrm{C}^{11} \mathrm{H}\right)$, $4.70\left(2 \mathrm{H}, \mathrm{s}, \mathrm{C}^{2} \mathrm{H}_{2}\right)^{*}, 4.65\left(2 \mathrm{H}, \mathrm{s}, \mathrm{C}^{11} \mathrm{H}_{2}\right)^{*}, 4.51\left(1 \mathrm{H}, \mathrm{s}, \mathrm{C}^{11} \mathrm{H}\right), 4.46$ $\left(4 \mathrm{H}, \mathrm{m}, \mathrm{C}^{17} \mathrm{H}_{2}, \mathrm{C}^{22} \mathrm{H}_{2}\right), 4.45\left(2 \mathrm{H}, \mathrm{s}, \mathrm{C}^{9} \mathrm{H}_{2}\right), 4.40\left(2 \mathrm{H}, \mathrm{s}, \mathrm{C}^{4} \mathrm{H}_{2}\right), 3.76$ $\left(4 \mathrm{H}, \mathrm{m}, \mathrm{C}^{16} \mathrm{H}_{2}, \mathrm{C}^{23} \mathrm{H}_{2}\right), 3.57\left(3 \mathrm{H}, \mathrm{s}, \mathrm{OCH}_{3}\right), 3.50\left(4 \mathrm{H}, \mathrm{s}, \mathrm{C}^{20} \mathrm{H}_{2}, \mathrm{C}^{19} \mathrm{H}_{2}\right)$, $2.39\left(2 \mathrm{H}, \mathrm{m}, \mathrm{C}^{10^{\prime}} \mathrm{H}, \mathrm{C}^{7} \mathrm{H}\right), 2.12\left(2 \mathrm{H}, \mathrm{m}, \mathrm{C}^{2} \mathrm{H}, \mathrm{C}^{10^{\prime}} \mathrm{H}\right), 1.94(1 \mathrm{H}, \mathrm{d} . \mathrm{m}$, $\left.J=12.1 \mathrm{~Hz}, \mathrm{C}^{8} \mathrm{H}\right), 1.86\left(1 \mathrm{H}\right.$, d.t, $\left.J=12.1 \mathrm{~Hz}, J=4.0 \mathrm{~Hz}, \mathrm{C}^{7} \mathrm{H}\right), 1.70-$ $1.78\left(3 \mathrm{H}, \mathrm{m}, \mathrm{C}^{\prime} \mathrm{H}, \mathrm{C}^{4} \mathrm{H}, \mathrm{C}^{3} \mathrm{H}\right), 1.67\left(1 \mathrm{H}, \mathrm{m}, \mathrm{C}^{9} \mathrm{H}\right), 1.60(2 \mathrm{H}, \mathrm{m}$, $\left.\mathrm{C}^{5} \mathrm{H}, \mathrm{C}^{9} \mathrm{H}\right), 1.45\left(1 \mathrm{H}, \mathrm{m}, \mathrm{C}^{3} \mathrm{H}\right), 1.21$ (1H, d.d, $J=12.0 \mathrm{~Hz}, J=2.7 \mathrm{~Hz}$, $\left.\mathrm{H}^{8 \mathrm{a}^{\prime}}\right), 1.13\left(3 \mathrm{H}, \mathrm{s}, \mathrm{C}^{13^{\prime}} \mathrm{H}_{3}\right), 0.97\left(1 \mathrm{H}\right.$, d.t, $\left.J=13.7 \mathrm{~Hz}, J=3.4 \mathrm{~Hz}, \mathrm{C}^{2} \mathrm{H}\right)$, $0.91\left(1 \mathrm{H}\right.$, d.t, $\left.J=13.7 \mathrm{~Hz}, J=4.0 \mathrm{~Hz}, \mathrm{C}^{4} \mathrm{H}\right), 0.44\left(3 \mathrm{H}, \mathrm{s}, \mathrm{C}^{14} \mathrm{H}_{3}\right) .{ }^{13} \mathrm{C}$ $\operatorname{NMR}\left(\mathrm{CDCl}_{3}, 298 \mathrm{~K}\right) \delta_{\mathrm{C}}$ ppm: $177.7\left(\mathrm{C}^{12}\right), 150.6\left(\mathrm{C}^{8}\right), 147.7\left(\mathrm{C}^{6}\right)$, $147.1\left(\mathrm{C}^{5}\right), 144.9\left(\mathrm{C}^{1}, \mathrm{C}^{12}\right), 125.2\left(\mathrm{C}^{6}\right), 123.8,123.7\left(\mathrm{C}^{28}, \mathrm{C}^{27}\right), 111.4$ $\left(\mathrm{C}^{7}\right), 106.4\left(\mathrm{C}^{11}\right), 69.2\left(\mathrm{C}^{17}, \mathrm{C}^{22}\right), 69.9\left(\mathrm{C}^{19}, \mathrm{C}^{20}\right), 64.6\left(\mathrm{C}^{9}\right), 64.1\left(\mathrm{C}^{11}\right)$, $63.9\left(\mathrm{C}^{2}\right), 62.3\left(\mathrm{C}^{4}\right), 56.0\left(\mathrm{C}^{8 \mathrm{a}^{\prime}}\right), 54.9\left(\mathrm{C}^{5}\right), 51.1\left(\mathrm{OCH}_{3}\right), 50.0\left(\mathrm{C}^{16}\right.$, $\left.\mathrm{C}^{23}\right), 44.2\left(\mathrm{C}^{1^{\prime}}\right), 40.1\left(\mathrm{C}^{4 \mathrm{a}^{\prime}}\right), 38.9\left(\mathrm{C}^{4}\right), 38.6\left(\mathrm{C}^{7}\right), 38.0\left(\mathrm{C}^{2}\right), 28.7$ $\left(\mathrm{C}^{13^{3}}\right), 26.2\left(\mathrm{C}^{8^{\prime}}\right), 24.4\left(\mathrm{C}^{9^{\prime}}\right), 23.1\left(\mathrm{C}^{10}\right), 19.8\left(\mathrm{C}^{3}\right), 12.6\left(\mathrm{C}^{14^{\prime}}\right)$.

Dimeric compound 23, oily substance. Found: C 62.98, H 7.82, $\mathrm{N} 12.67 \%$. [M] 1267. $\mathrm{C}_{70} \mathrm{H}_{100} \mathrm{~N}_{12} \mathrm{O}_{14}$. requires C 63.04, H 7.56, N 12.60\%. [M] 1332. UV (EtOH) $\lambda_{\text {max }} \mathrm{nm}(\lg \varepsilon): 221$ (4.05), 285 (2.76). IR $(\mathrm{KBr}) v \mathrm{~cm}^{-1}$ : $665 \mathrm{w}, 755 \mathrm{w}, 822 \mathrm{w}, 894 \mathrm{w}, 919 \mathrm{w}, 987 \mathrm{w}, 1052 \mathrm{~m}$, $1072 \mathrm{~m}, 1137 \mathrm{~m}, 1149 \mathrm{~m}, 1228 \mathrm{~m}, 1331 \mathrm{w}, 1354 \mathrm{w}, 1453 \mathrm{~m}, 1467 \mathrm{~m}$, 1553 m, 1645 w, 1720 s, 2870 m, 2946 m, 3080 s, 3143 s. ${ }^{1} \mathrm{H}$ NMR $\left(\mathrm{CDCl}_{3}, 298 \mathrm{~K}\right) \delta_{\mathrm{H}}$ ppm: 7.70, $7.70\left(2 \mathrm{H} \text {, all s, } 2 \mathrm{C}^{5 "} \mathrm{H}\right)^{*}, 7.68,7.68(2 \mathrm{H}$, all s, $\left.2 \mathrm{C}^{5} \mathrm{H}\right)^{*}, 6.18\left(2 \mathrm{H}, \mathrm{s}, 2 \mathrm{C}^{14} \mathrm{H}\right), 4.87\left(2 \mathrm{H}, \mathrm{s}, 2 \mathrm{C}^{17} \mathrm{H}\right), 4.61(4 \mathrm{H}, \mathrm{d}$, $\left.J=2.0 \mathrm{~Hz}, 2 \mathrm{C}^{4 \prime} \mathrm{CH}_{2}\right)^{*}, 4.58\left(4 \mathrm{H}, \mathrm{d}, J=2.0 \mathrm{~Hz}, 2 \mathrm{C}^{4} \mathrm{CH}_{2}\right)^{*}, 4.53(2 \mathrm{H}, \mathrm{s}$, $\left.2 \mathrm{C}^{17} \mathrm{H}\right), 4.44\left(8 \mathrm{H}, \mathrm{m}, 4 \mathrm{CH}_{2} \mathrm{O}\right), 4.41\left(4 \mathrm{H}, \mathrm{s}, 2 \mathrm{C}^{15} \mathrm{CH}_{2}\right) *, 4.39(4 \mathrm{H}, \mathrm{s}$, $\left.2 \mathrm{C}^{16} \mathrm{CH}_{2}\right), 3.75\left(8 \mathrm{H}, \mathrm{m}, 2 \mathrm{CH}_{2} \mathrm{~N}^{1}, 2 \mathrm{CH}_{2} \mathrm{~N}^{1 "}\right), 3.59\left(6 \mathrm{H}, \mathrm{s}, 2 \mathrm{OCH}_{3}\right), 3.49$ $\left(8 \mathrm{H}, \mathrm{s}, 2 \mathrm{OCH}_{2} \mathrm{CH}_{2} \mathrm{O}\right), 2.49\left(2 \mathrm{H}, \mathrm{m}, 2 \mathrm{C}^{12} \mathrm{H}\right), 2.39(2 \mathrm{H}, \mathrm{d} . \mathrm{m}, J=11.6 \mathrm{~Hz}$ $\left.2 \mathrm{C}^{7} \mathrm{H}\right), 2.20\left(2 \mathrm{H}, \mathrm{m}, 2 \mathrm{C}^{12} \mathrm{H}\right), 2.12\left(2 \mathrm{H}\right.$, d.m, $\left.J=13.2 \mathrm{~Hz}, 2 \mathrm{C}^{3} \mathrm{H}\right), 1.95$ $\left(2 \mathrm{H}, \mathrm{m}, 2 \mathrm{C}^{6} \mathrm{H}\right), 1.82\left(2 \mathrm{H}, \mathrm{m}, J=3.2 \mathrm{~Hz}, 2 \mathrm{C}^{7} \mathrm{H}\right), 1.73-1.77(6 \mathrm{H}$, all m, $\left.2 \mathrm{C}^{1} \mathrm{H}, 2 \mathrm{C}^{2} \mathrm{H}, 2 \mathrm{C}^{6} \mathrm{H}\right), 1.67\left(2 \mathrm{H}, \mathrm{m}, 2 \mathrm{C}^{11} \mathrm{H}\right), 1.55\left(4 \mathrm{H}, \mathrm{m}, 2 \mathrm{C}^{9} \mathrm{H}, 2 \mathrm{C}^{11} \mathrm{H}\right)$, $1.49\left(2 \mathrm{H}, \mathrm{m}, 2 \mathrm{C}^{2} \mathrm{H}\right), 1.26\left(2 \mathrm{H}, \mathrm{d} . \mathrm{d}, J=12.0 \mathrm{~Hz}, J=2.6 \mathrm{~Hz}, 2 \mathrm{C}^{5} \mathrm{H}\right), 1.15$ $\left(6 \mathrm{H}, \mathrm{s}, 2 \mathrm{C}^{19} \mathrm{H}_{3}\right), 0.99\left(2 \mathrm{H}\right.$, d.t, $\left.J=13.0 \mathrm{~Hz}, J=3.6 \mathrm{~Hz}, 2 \mathrm{C}^{3} \mathrm{H}\right), 0.96(2 \mathrm{H}$, $\left.\mathrm{m}, 2 \mathrm{C}^{1} \mathrm{H}\right), 0.46\left(6 \mathrm{H}, \mathrm{s}, 2 \mathrm{C}^{20} \mathrm{H}_{3}\right) \cdot{ }^{13} \mathrm{C} \mathrm{NMR}\left(\mathrm{CDCl}_{3}, 298 \mathrm{~K}\right) \delta_{\mathrm{C}} \mathrm{ppm}$ : $177.7\left(2 \mathrm{C}^{18}\right), 150.7\left(2 \mathrm{C}^{15}\right), 147.7\left(2 \mathrm{C}^{8}\right), 147.13,147.16\left(2 \mathrm{C}^{16}\right), 144.68$, $144.72,144.51,144.56\left(2 \mathrm{C}^{4}, 2 \mathrm{C}^{4 \prime}\right), 125.4\left(2 \mathrm{C}^{13}\right), 123.9,123.8\left(2 \mathrm{C}^{5^{\prime}}\right.$ $\left.2 \mathrm{C}^{5 "}\right), 111.62,111.64\left(2 \mathrm{C}^{14}\right), 106.4\left(2 \mathrm{C}^{17}\right), 70.2\left(2 \mathrm{OCH}_{2} \mathrm{CH}_{2} \mathrm{O}\right), 69.3$ $\left(4 \mathrm{CH}_{2} \mathrm{O}\right), 64.3,64.4\left(2 \mathrm{CH}_{2} \mathrm{C}^{15}\right), 63.62,63.65,63.54,63.57\left(2 \mathrm{CH}_{2} \mathrm{C}^{4}\right.$, $\left.2 \mathrm{CH}_{2} \mathrm{C}^{41}\right), 62.2,62.3\left(2 \mathrm{CH}_{2} \mathrm{C}^{16}\right), 56.1\left(2 \mathrm{C}^{5}\right), 55.1\left(2 \mathrm{C}^{9}\right), 51.1\left(2 \mathrm{OCH}_{3}\right)$, $50.03,50.05\left(2 \mathrm{CH}_{2} \mathrm{~N}^{1}, 2 \mathrm{CH}_{2} \mathrm{~N}^{1}\right), 44.2\left(2 \mathrm{C}^{4}\right), 40.2\left(2 \mathrm{C}^{10}\right), 39.0\left(2 \mathrm{C}^{1}\right)$, $38.7\left(2 \mathrm{C}^{7}\right), 38.1\left(2 \mathrm{C}^{3}\right), 28.8\left(2 \mathrm{C}^{19}\right), 26.2\left(2 \mathrm{C}^{6}\right), 24.6\left(2 \mathrm{C}^{11}\right), 23.3\left(2 \mathrm{C}^{12}\right)$, $19.9\left(2 \mathrm{C}^{2}\right), 12.6\left(2 \mathrm{C}^{20}\right)$.

Trimeric compound 24 , oily substance. Found: C 62.93, H 7.34, $\mathrm{N}$ 12.35\%. [M] 1893. $\mathrm{C}_{105} \mathrm{H}_{150} \mathrm{~N}_{18} \mathrm{O}_{21}$. requires C 63.04, H 7.56, N 12.60\%. [M] 1998. UV (EtOH) $\lambda_{\max } \mathrm{nm}(\lg \varepsilon): 221$ (4.03), 286 (2.74) $\mathrm{IR}(\mathrm{KBr}) v \mathrm{~cm}^{-1}: 667 \mathrm{w}, 755 \mathrm{w}, 822 \mathrm{w}, 894 \mathrm{w}, 920 \mathrm{w}, 990 \mathrm{w}, 1049 \mathrm{~m}$, $1071 \mathrm{~m}, 1137 \mathrm{~m}, 1149 \mathrm{~m}, 1228 \mathrm{~m}, 1334 \mathrm{w}, 1358 \mathrm{w}, 1451 \mathrm{~m}, 1465 \mathrm{~m}$, 1555 m, 1644 w, 1721 s, 2870 m, 2946 m, 3078 s, 3143 s. ${ }^{1} \mathrm{H}$ NMR $\left(\mathrm{CDCl}_{3}, 298 \mathrm{~K}\right) \delta_{\mathrm{H}} \mathrm{ppm}: 7.55,7.65,7.67\left(6 \mathrm{H}\right.$, all s, 3C $\left.\mathrm{C}^{5} \mathrm{H}, 3 \mathrm{C}^{5 \prime} \mathrm{H}\right), 6.19$ $\left(3 \mathrm{H}, \mathrm{s}, 3 \mathrm{C}^{14} \mathrm{H}\right), 4.84\left(3 \mathrm{H}, \mathrm{s}, 3 \mathrm{C}^{17} \mathrm{H}\right), 4.61\left(6 \mathrm{H}, \mathrm{s}, 3 \mathrm{C}^{4 \prime} \mathrm{CH}_{2}\right)^{*}, 4.57(6 \mathrm{H}$, $\left.\mathrm{s}, 3 \mathrm{C}^{4} \mathrm{CH}_{2}\right)^{*}, 4.51\left(3 \mathrm{H}, \mathrm{s}, 3 \mathrm{C}^{17} \mathrm{H}\right), 4.43\left(18 \mathrm{H}, \mathrm{s}, 3 \mathrm{C}^{15} \mathrm{CH}_{2}, 6 \mathrm{CH}_{2} \mathrm{O}\right)$, $4.39\left(6 \mathrm{H}, \mathrm{s}, 3 \mathrm{C}^{16} \mathrm{CH}_{2}\right), 3.76\left(12 \mathrm{H}, \mathrm{s}, 3 \mathrm{CH}_{2} \mathrm{~N}^{1}, 3 \mathrm{CH}_{2} \mathrm{~N}^{1 \prime}\right), 3.58(9 \mathrm{H}$, $\left.\mathrm{s}, 3 \mathrm{OCH}_{3}\right), 3.49\left(12 \mathrm{H}, \mathrm{s}, 3 \mathrm{OCH}_{2} \mathrm{CH}_{2} \mathrm{O}\right), 2.47\left(3 \mathrm{H}, \mathrm{m}, 3 \mathrm{C}^{12} \mathrm{H}\right), 2.36$ ( $3 \mathrm{H}$, d.m, $\left.J=10.8 \mathrm{~Hz}, 3 \mathrm{C}^{7} \mathrm{H}\right), 2.19\left(3 \mathrm{H}, \mathrm{m}, 3 \mathrm{C}^{12} \mathrm{H}\right), 2.10$ (3H, d.m, $\left.J=13.4 \mathrm{~Hz}, 3 \mathrm{C}^{3} \mathrm{H}\right), 1.94\left(3 \mathrm{H}, \mathrm{m}, 3 \mathrm{C}^{6} \mathrm{H}\right), 1.83\left(3 \mathrm{H}, \mathrm{m}, 3 \mathrm{C}^{7} \mathrm{H}\right), 1.71-$ $1.78\left(9 \mathrm{H}, \mathrm{m}, 3 \mathrm{C}^{1} \mathrm{H}, 3 \mathrm{C}^{2} \mathrm{H}, 3 \mathrm{C}^{6} \mathrm{H}\right), 1.65\left(3 \mathrm{H}, \mathrm{m}, 3 \mathrm{C}^{11} \mathrm{H}\right), 1.54(6 \mathrm{H}$, $\left.\mathrm{m}, 3 \mathrm{C}^{9} \mathrm{H}, 3 \mathrm{C}^{11} \mathrm{H}\right), 1.45\left(3 \mathrm{H}, \mathrm{m}, 3 \mathrm{C}^{2} \mathrm{H}\right), 1.24(3 \mathrm{H}$, d.d, $J=12.0 \mathrm{~Hz}$, $\left.J=2.2 \mathrm{~Hz}, 3 \mathrm{C}^{5} \mathrm{H}\right), 1.13\left(9 \mathrm{H}, \mathrm{s}, 3 \mathrm{C}^{19} \mathrm{H}_{3}\right), 0.97(3 \mathrm{H}$, d.t, $J=12.9 \mathrm{~Hz}$, $\left.J=3.2 \mathrm{~Hz}, 3 \mathrm{C}^{3} \mathrm{H}\right), 0.94\left(3 \mathrm{H}\right.$, d.t, $\left.J=12.9 \mathrm{~Hz}, J=2.7 \mathrm{~Hz}, 3 \mathrm{C}^{1} \mathrm{H}\right), 0.44$ $\left(9 \mathrm{H}, \mathrm{s}, 3 \mathrm{C}^{20} \mathrm{H}_{3}\right) \cdot{ }^{13} \mathrm{C} \mathrm{NMR}\left(\mathrm{CDCl}_{3}, 298 \mathrm{~K}\right) \delta_{\mathrm{C}} \mathrm{ppm}: 177.66,177.68$, $177.70\left(3 \mathrm{C}^{18}\right), 150.67,150.69,150.72\left(3 \mathrm{C}^{15}\right), 147.81,147.84\left(3 \mathrm{C}^{8}\right)$, 147.05, 147.06, $147.09\left(3 \mathrm{C}^{16}\right), 144.7,144.8,144.6,144.7\left(3 \mathrm{C}^{4}\right.$, $\left.3 \mathrm{C}^{4 \prime}\right), 125.43,125.45\left(3 \mathrm{C}^{13}\right), 123.8,123.9,123.6,123.7\left(3 \mathrm{C}^{5}, 3 \mathrm{C}^{5 "}\right)$, $111.62,111.64,111.65\left(3 \mathrm{C}^{14}\right), 106.3\left(3 \mathrm{C}^{17}\right), 70.3\left(3 \mathrm{OCH}_{2} \mathrm{CH}_{2} \mathrm{O}\right), 69.3$ $\left(6 \mathrm{CH}_{2} \mathrm{O}\right), 64.26,64.29\left(3 \mathrm{CH}_{2} \mathrm{C}^{15}\right), 63.39,63.40,63.42,63.27,63.29$, $63.30\left(3 \mathrm{CH}_{2} \mathrm{C}^{4}, 3 \mathrm{CH}_{2} \mathrm{C}^{4 \prime}\right), 62.21,62.24,62.25\left(3 \mathrm{CH}_{2} \mathrm{C}^{16}\right), 56.0\left(3 \mathrm{C}^{5}\right)$, $55.0\left(3 \mathrm{C}^{9}\right), 51.1\left(3 \mathrm{OCH}_{3}\right), 50.1,50.05\left(\mathrm{t}, 3 \mathrm{CH}_{2} \mathrm{~N}^{1}, 3 \mathrm{CH}_{2} \mathrm{~N}^{1 "}\right), 44.2$ $\left(3 \mathrm{C}^{4}\right), 40.1\left(3 \mathrm{C}^{10}\right), 38.9\left(3 \mathrm{C}^{1}\right), 38.6\left(3 \mathrm{C}^{7}\right), 38.0\left(3 \mathrm{C}^{3}\right), 28.2\left(3 \mathrm{C}^{19}\right), 26.2$ $\left(3 \mathrm{C}^{6}\right), 24.5\left(3 \mathrm{C}^{11}\right), 23.3\left(3 \mathrm{C}^{12}\right), 19.8\left(3 \mathrm{C}^{2}\right), 12.5\left(3 \mathrm{C}^{20}\right)$.

\section{Results and Discussion}

The synthetic route followed for the synthesis of the key compound - labdanoid diacetylenic derivatives $\mathbf{1 1}$ is outlined in Scheme 1. Vilsmeier-Haack formylation of compound $\mathbf{3}$ gave its 15 -formyl derivative $\mathbf{8}$ which was converted to the compound 9 by treatment with sodium borohydride in $i$-propanol. By reduction of $\mathbf{8}$ in methanol the compound $\mathbf{1 0}$ was obtained exclusively. The reaction of 15-hydroxymethyl labdatrinoate 9 with propargyl bromide in DMF in the presence of sodium hydride resulted in formation of diacetylenic derivatives 11 (yield $34 \%$ ) and compound 12 (yield $23 \%$ ). Compound $\mathbf{1 1}$ was obtained in $75 \%$ yield by reaction of compound 9 with propargyl bromide in acetonitrile.

The terpenoid dialkyne $\mathbf{1 1}$ was reacted with 1 equivalent of 1,5-diazidopentane 4 in $\mathrm{CH}_{2} \mathrm{Cl}_{2}$-water medium (20:1; $0.05 \mathrm{M}$ solution of 11) in the presence of $\mathrm{CuSO}_{4}$ and sodium ascorbate in conditions used in our previous studies. ${ }^{[2]}$ By performing the reaction at $40{ }^{\circ} \mathrm{C}$ over $10 \mathrm{~h}$ the full conversion of compound $\mathbf{1 1}$ was observed. After column chromatography on silica gel four compounds were isolated: diazide 13 (7\%), macroheterocyclic compound 14 (13\%), cyclic dimer $15(42 \%)$ and cyclic trimer 


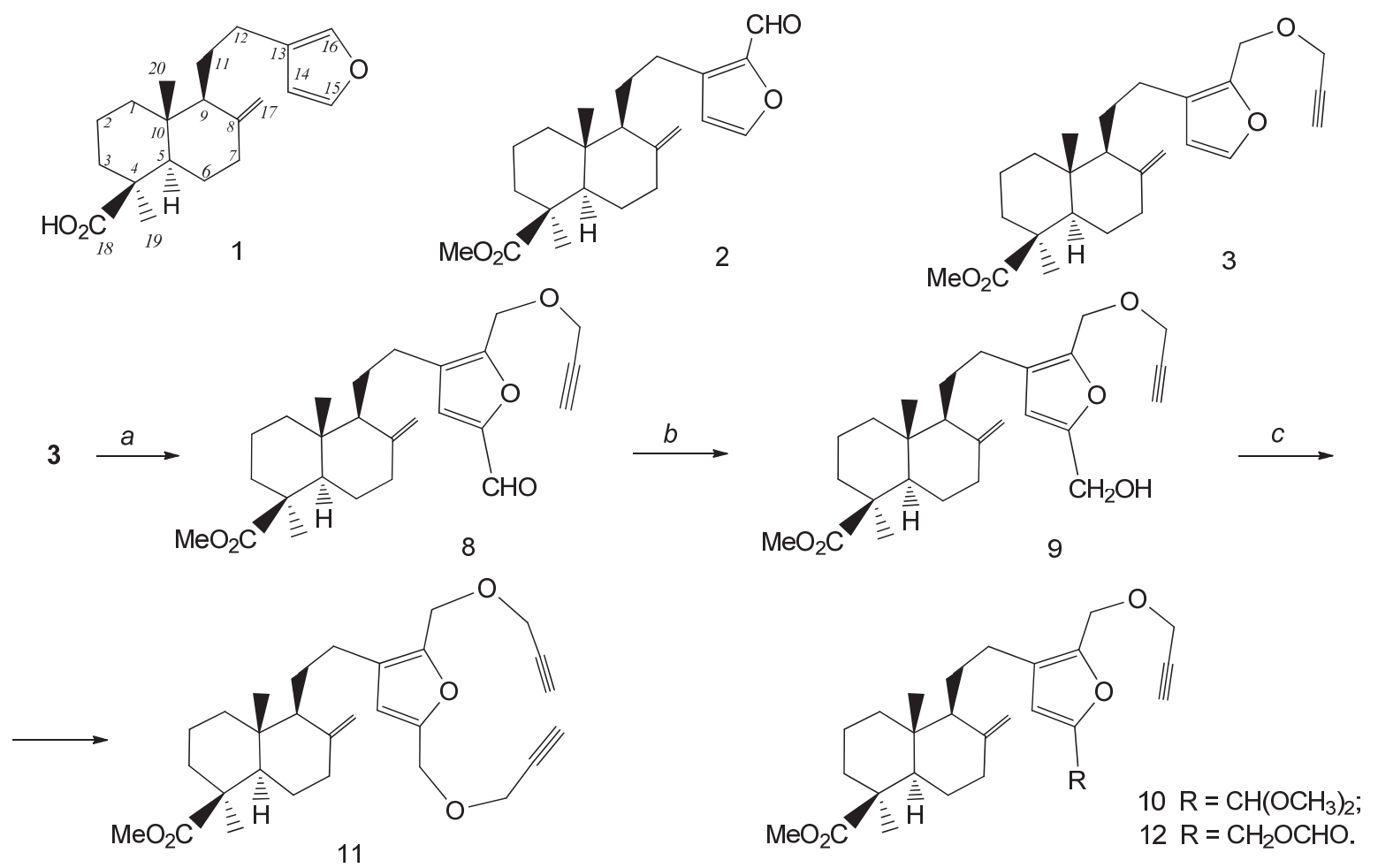

Reagents and Reaction Conditions: (a): $\mathrm{POCl}_{3}$, DMF, AcONa, $20^{\circ} \mathrm{C}, 48 \mathrm{~h}$; (b): $\mathrm{NaBH}_{4}$, i-PrOH, $20^{\circ} \mathrm{C}, 24 \mathrm{~h}$; (c) $\mathrm{BrCH}_{2} \mathrm{C} \equiv \mathrm{CH}$, $\mathrm{NaH}, \mathrm{CH}_{3} \mathrm{CN}, 0^{\circ} \mathrm{C}$, then rt, $4 \mathrm{~h}$.

\section{Scheme 1.}

$16(20 \%)$ (Scheme 2). Dilution of the reaction mixture with methylene chloride ( $0.01 \mathrm{M}$ solution) improved the yield of the target compound 14 to $68 \%$. Additionally, dimeric compound 15 was also isolated in the yield $12 \%$.

Reaction of dialkyne 11 ( $0.01 \mathrm{M}$ solution in methylene chloride) with 1,10-diazidodecane (5) in the presence of $\mathrm{CuSO}_{4}$ and sodium ascorbate resulted in the formation of a mixture of compound $\mathbf{1 7}$ and dimeric compound $\mathbf{1 8}$ isolated in $53 \%$ and $30 \%$ yield after column chromatography.

By using of the mentioned CuAAC conditions for reacting of diterpenoid dialkyne 11 with 1-azido-2-(2azidoethoxy)ethane $\mathbf{6}$ the compound $\mathbf{1 9}$ was isolated in the yield $38 \%$ (Scheme 3). Cyclodimer 20 and cyclotrimer 21 were also isolated in the yield $22 \%$ and $26 \%$ respectivelly.

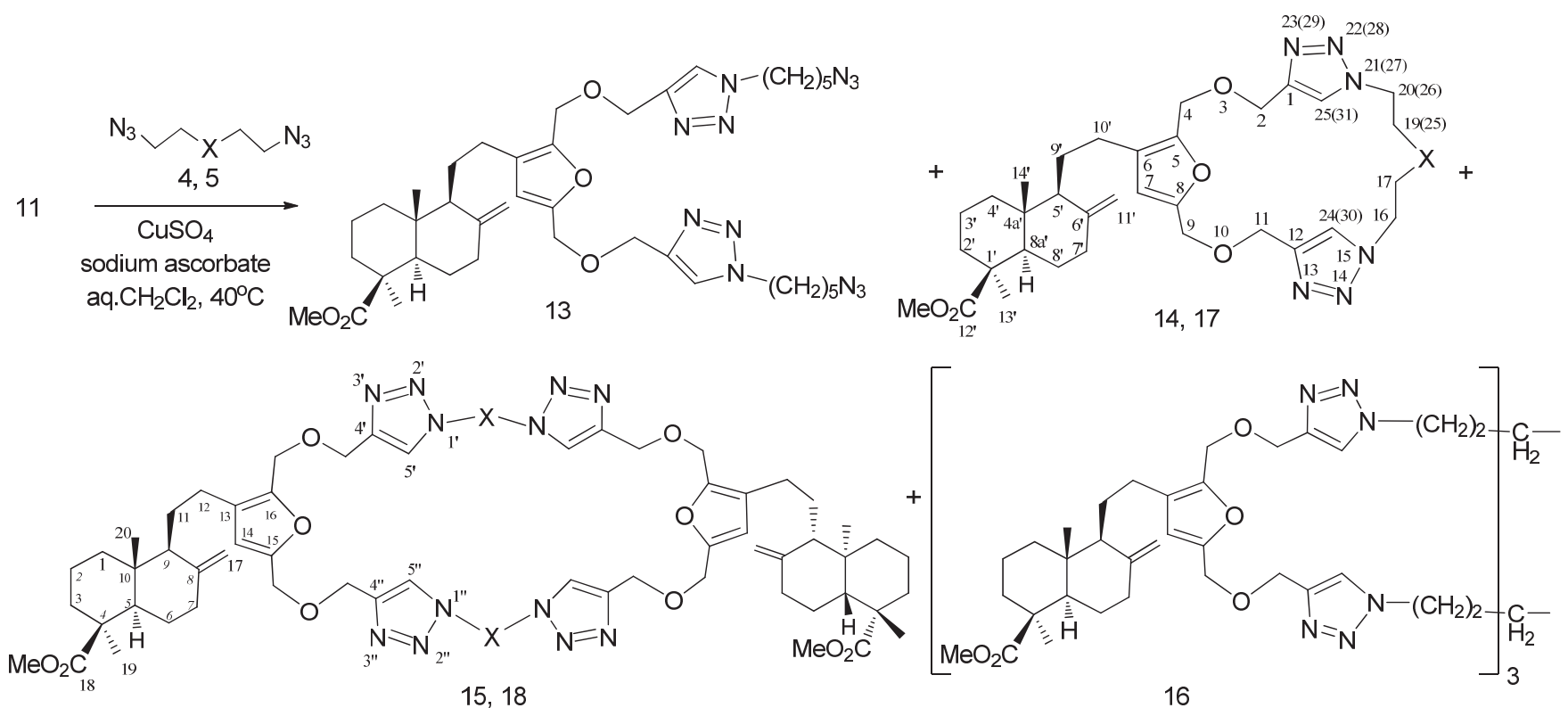

$\mathrm{X}=\mathrm{C}^{18} \mathrm{H}_{2}(\mathbf{4}, 14,15) ; \mathrm{CH}_{2}^{(19)} \mathrm{CH}_{2}^{(20)} \mathrm{CH}_{2}^{(21)} \mathrm{CH}_{2}^{(22)} \mathrm{CH}_{2}^{(23)} \mathrm{CH}_{2}^{(24)}(5,17,18)$ 


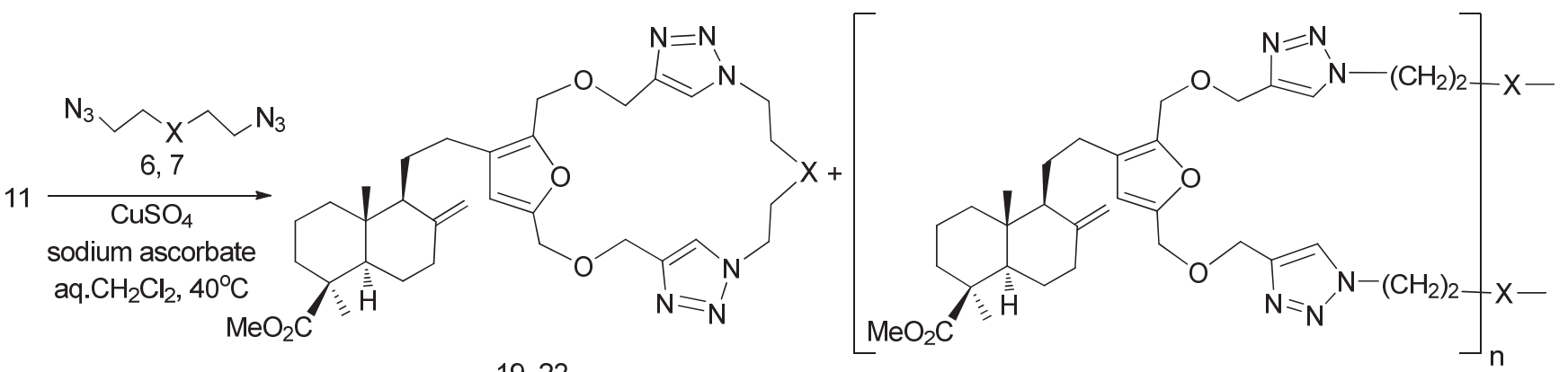

19,22

$20,21,23,24$

$X=O(6,19,20,21) ; \mathrm{O}\left(\mathrm{CH}_{2}\right)_{2} \mathrm{O}(7,22,23,24) ; \mathrm{n}=2(20,23) ; 3(21,24)$.

Scheme 3 .

Comparable results were obtained in the reaction of dialkyne 11 with 1,2-bis(2-azidoethoxy)ethane (7); macrocyclic derivatives 22, 23 and $\mathbf{2 4}$ were obtained in the yield of $32 \%, 10 \%$ and $31 \%$ after column chromatography.

The composition and structure of the synthesized compounds were confirmed by IR, UV, ${ }^{1} \mathrm{H}$, and ${ }^{13} \mathrm{C}$ spectroscopy, mass-spectrometry, elemental analysis data and mass-date for dimeric and trimeric compounds. The ${ }^{1} \mathrm{H}$ and ${ }^{13} \mathrm{C}$ NMR spectra of all synthesized compounds agree with their structure and contain the set of characteristic signals of labdanoid skeleton and the corresponding substituent. Formation of the 1,2,3-triazole ring in compounds 13-24 was confirmed by the NMR data. The 'H NMR spectra exhibited singlet signals for the H-5' proton $(\delta=7.37-7.68 \mathrm{ppm})$. The ${ }^{13} \mathrm{C}$ NMR signals of the $\mathrm{C} 4-$ ', 5 ' carbon atoms were observed in the region of 144.6-145.9 ppm and 122.3-124.0 ppm, respectively. Macrocyclic 14, 19 and cyclodimeric compounds 15, 20 with a 5-membered linker between triazole rings possess greatly different in the shifts of the $\mathrm{H}-5$ proton in the triazole ring. A shift around $0.2 \mathrm{ppm}$ was observed for the signal of $\mathrm{H}-5$ atom in the ${ }^{1} \mathrm{H}$ NMR spectra of compound 15, 20 compared with compounds 14, 19.

\section{Conclusions}

As a result, we have elaborated bi-, tetra- and hexa(1,2,3-triazol)containing furan bridged macrocyclic compounds using CuAAC reaction of diacetylenic derivatives of easily available methyl lambertianate with various diazides. The yield and composition of the target macrocyclic compounds were shown to be dependent on the nature of the starting diazides, better yield of bi(1,2,3-triazol)containing furan bridged macrocyclic compound being observed in the case of 1,5-diazidopentane and 1,10-diazidodecane.

Acknowledgements. This work was supported in part by the Grants Council of the president of the Russian Federation (NS-2625.2014.3) and Russian Science Foundation (Grant Nom 14-13-00822).

\section{References}

1. Gale P.A., Gunnlaugsson T. Chem. Soc. Rev. 2010, 39, 35814008.
2. Lee J.W., Samal S., Selvapalam N., Kim H.-J., Kim K. Acc. Chem. Res. 2003, 36, 621-630.

3. Marsault E., Peterson M.L. J. Med. Chem. 2011, 54, 19612004.

4. Virtanen E., Kolehmainen E. Eur. J. Org. Chem. 2004, 33853399.

5. Cho H., Widanapathirana L., Zhao Y. J. Am. Chem. Soc. 2011, 133, 141-147.

6. Bag B.G., Dinda S.K., Dey P.P., Mallia V.A., Weiss R.G. Langmuir 2009, 25, 8663-8671.

7. Hu J., Li R., Lu J., Ju Y. Tetrahedron Lett. 2011, 52, 42114214.

8. Hu J., Wu J., Lu J., Ju Y. Tetrahedron Lett. 2012, 53, 6705-6709.

9. Chattopadhyay P., Pandey P.S. Tetrahedron 2006, 62, 86208624.

10. Juráŝek M., Džubák P., Sedlák D., Dvořáková H., Hajdúch M., Bartuněk P., Drašar P. Steroids 2013, 78, 356-361.

11. Hu J., Zhang M., Yu L. B., Ju Y. Bioorg. Med. Chem. Lett. 2010, 20, 4342-4346.

12. Kataev V.E., Khaybullin R.N., Sharipova R.R., Strobykina I.Yu. Rev. J. Chem. 2011, 1, 93-160.

13. Andreeva O.A., Strobykina I.Yu., Kataeva O.N., Dobrynin A.B., Babaev V.M., Rizvanov I.Kh., Islamov D.R., Kataev V.E. Macroheterocycles 2013, 6, 315-322.

14. Khaybullin R.N., Strobykina I.Yu., Dobrynin A.B., Gubaydullin A.T., Chestnova R.V., Babaev V.M., Kataev V.E. Bioorg. Med. Chem. Lett. 2012, 22, 6909-6913.

15. Ojima I., Das M. J. Nat. Prod. 2009, 72, 554-565.

16. Meldal M., Tornoe C.W. Chem. Rev. 2008, 108, 2952-3015.

17. Kharitonov Yu.V., Shul'ts E.E., Shakirov M.M., Bagryanskaya I.Yu., Tolstikov G.A. Doklady Chemistry 2012, 446, 174-179.

18. Blunt J.W., Copp B.R., Keyzers R.A., Munro M.H.G., Prinsep M.R. Nat. Prod. Rep. 2013, 30, 237-323.

19. Patent RF 2460741 Invention Bull. 2012, N 9 (in Russ.).

20. Klok D.A., Shakirov M.M., Grishko V.V., Raldugin V.A. Russ. Chem. Bull. (Engl. Transl.) 1995, 44, 2412-2414 [Izv. Akad. Nauk, Ser. Khim. 1995, 2514-2517 (in Russ.)].

21. Saikia B., Saikia P.P., Goswami A., Barua N.C., Saxena A.K., Suri N. Synthesis 2011, 3173-3179.

22. Katrytzky A.R., Meher N.K., Hanci S., Gyanda R., Tala S.R., Mathai S., Duran R.S., Bernard S., Sabri F., Singh S.K., Doskocz J., Ciaramitaro D.A. J. Polymer Sci. A, Pol. Chem. 2008, 46, 238-256.

23. Klein E., DeBonis S., Thiede B., Skoufias D.A., Kozielski F., Lebeau L. Bioorg. Med. Chem. 2007, 15, 6474-6488.

24. Kharitonov Yu.V., Shul'ts E.E., Shakirov M.M., Pokrovsky M.A., Pokrovsky A.G., Tolstikov G.A. Russ. Chem. Bull. 2013, 62, 2046-2055.

Received 12.11.2014 Accepted 25.11.2014 